\title{
EL ESLIZON IBERICO (Chalcides Bedriagai) EN EL MEDIO INSULAR DE NUEVA TABARCA (Provincia de Alicante)
}

Por E. SEVA* y A. ESCARRE**

\section{S U M A R I O}

Página

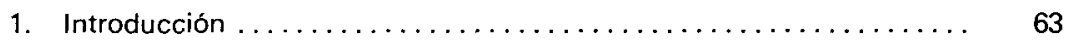

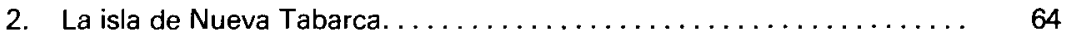

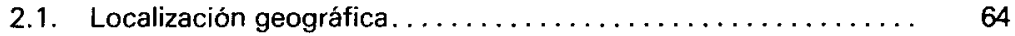

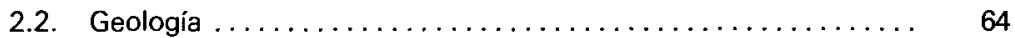

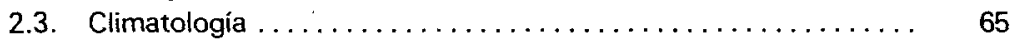

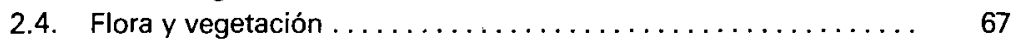

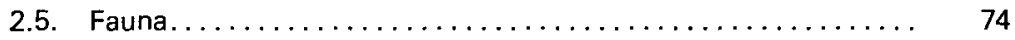

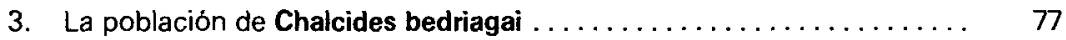

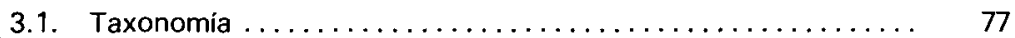

3.1.1. Sistemática del eslizón ibérico .............. 77

3.1.2. Definición de caracteres ................. 80

3.1.3. Ejemplares estudiados................... 84

3.1.4. Modelo de correlación de los caracteres. .......... 87

3.1.5. Diagnosis subespecífica $\ldots \ldots \ldots \ldots \ldots \ldots \ldots \ldots \ldots . \ldots \ldots$

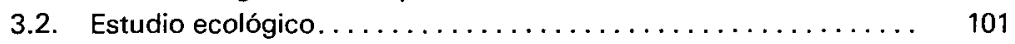

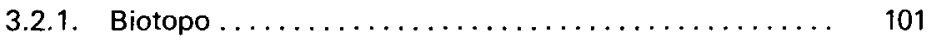

3.2.2. Estructura de la población ................ 103

3.2.3. Alimentación. Análisis de contenidos estomacales. Retículo trófico ....................... 106

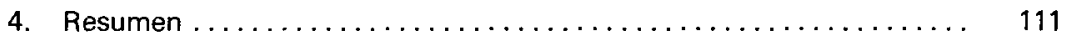

5. Bibliografía.................................. 113

* Departamento de Biología y Geología, C.E.U., Alicante.

** Idem. (Dirección actual: Departamento de Ecología, Facultad de Biología, Barcelona). 



\section{1) INTRODUCCION}

La elección de este trabajo responde al interés que presenta el fenómeno del aislamiento insular, interés que ha suscitado, para las islas mediterráneas más próximas al litoral español, trabajos como los de ESPAÑOL (1965) sobre la fauna entomológica de este mismo archipiélago $y$ el de BALCELLS (1963) sobre el poblamiento vegetal $y$ animal de las islas Medas.

Si bien al principio se pensó hacer un estudio global de los vertebrados de este medio insular, la superabundancia de Chalcides bedriagai unida al interés sistemático que presentan las subespecies que escuetamente describió VALVERDE (1966), dirigió nuestra atención hacia la posibilidad de aportar nuevos datos de este endemismo ibérico.

El estudio del medio insular de N. Tabarca ha sido emprendido desde dos aspectos. En la primera parte del trabajo se han reunido los datos que hacen referencia a la geología, climatología, flora y vegetación y fauna de las islas. La segunda parte tiene como objetivo específico el estudio de la población de Chalcides bedriagai; desde el punto de vista taxonómico se trata de abordar el problema sistemático del eslizón mediante un análisis estadístico de los caracteres, que permiten una diagnosis subespecífica. El aspecto ecológico se centra en el estudio de la estructura de la población, alimentación y un esbozo del retículo trófico donde se halla integrado.

En el apartado de agradecimientos se incluyen: J. R. VERICAD, J. M. REY y J. P. MARTINEZ-RICA, por las facilidades que dieron en la consulta de colecciones; N. SAULEDA, por la identificación de gran parte del material entomológico; P. GARRIDO, por su información sobre el programa CHARANAL; y F. JIMENEZ, A. POVEDA, A. LASPALAS, M. BENITEZ, M. R. VIDAL y L. VILLACORTA, por sus sugerencias y la ayuda prestada en distintos aspectos y etapas del trabajo.

Finalmente también se hace extensivo el agradecimiento a todos los pobladores de Nueva Tabarca que han proporcionado interesante información sobre el medio insular que habitan. 


\section{2) LA ISLA DE NUEVA TABARCA}

\subsection{Localización geográfica}

El archipiélago de Nueva Tabarca se halla situado en el litoral levantino a quince kilómetros al sur de la ciudad de Alicante $y$ al frente mismo del cabo de Santa Pola, del que dista cuatro kilómetros. Las coordenadas UTM son: YH-(19 a 23)-27.

Sus componentes son: la isla Plana o Nueva Tabarca, proyectada según la dirección NO-SE, de forma alargada y con un estrechamiento en su tercio oeste, con tres islotes con los nombres de La Cantera, La Galera y La Nao situados respectivamente al oeste, sur y este de la misma. La longitud aproximada de Isla Plana es de $1.800 \mathrm{~m}$., y su anchura máxima, de $400 \mathrm{~m}$. La profundidad media de los alrededores de la isla es de unos siete metros, cifra que aumenta en la parte este. Al norte del estrechamiento antes citado se alberga un pequeño puerto, $y$, al sur, la única playa arenosa de la isla, ya que la línea de costa la forman pequeños acantilados de pocos metros.

La población está formada por alrededor de doscientos habitantes, que se dedican principalmente a la pesca y al turismo, y se concentran en el poblado de $S$. Pablo, situado en el extremo noroeste y que está totalmente rodeado de unas antiguas murallas que datan del reinado de Carlos III.

En la parte más ancha de la isla se sitúan de forma esparcida tres construcciones: la torre de S. José, que es una antigua fortaleza de gruesas paredes actualmente convertida en cuartel de la Guardia Civil; una vieja casa de campo en ruinas hacia el centro; y más hacia el extremo oriental, antes de llegar al cementerio, el nuevo faro, construido junto al antiguo. Sin duda lo que más llama la atención es el extraordinario aplanamiento de esta parte de la isla, en la que todavia quedan restos de unos antiguos cultivos abandonados.

La ausencia total de arbolado hace que se carezca de protección contra el sol del verano y los periódicos y fuertes vientos de levante.

\subsection{Geología}

La bibliografía existente hasta el momento sobre la geología de Nueva Tabarca se reduce al estudio de KAMPSCHUUR Y SIMON (1969) y a los datos que en su tesis incluye MONTENAT (1973). 
De las tres grandes zonas en que tradicionalmente se han dividido las cordilleras Béticas (Prebética, Subbética y Bética) la isla de Nueva Tabarca corresponde a la zona Bética y es su afloramiento más oriental conocido.

Respecto a su estratigrafia, los materiales preorogénicos están constituidos por calizas y dolomías, yesos y carniolas, que constituyen la serie Bética (KAMPSCHUUR Y SIMON, 1969). Además afloran rocas ígneas, principalmente metabasitas.

El Mioceno, discordante y suprayacente a los términos anteriores, aparece como un conjunto de materiales constituidos por calizas arenosas amarillas, bastante gruesas y muy. fosiliferas, con Hesterostegina, Briozoarios, Pectínidos, Ostrea y Equínidos. Atribuye MONTENAT (1973) a este conjunto la edad Tortoniense Superior por correlación de facies con sus equivalentes de Alicante y Elche. El espesor aproximado de esta formación es de cien metros.

El Cuaternario aparece sobre los terrenos descritos, como un conjunto de materiales que MONTENAT atribuye a la edad Tirreniense $y$ que están constituidos por conglomerados, cuyos cantos de tamaño 0,4 metros son fundamentalmente rocas verdes. Alcanzan hacia el borde sur de la isla un espesor variable de uno a dos metros.

\subsection{Climatología}

La falta de datos meteorológicos de Nueva Tabarca ha obligado al estudio del entorno climático más próximo y en él se han elegido las estaciones de Alicante (Ciudad Jardín) y Guardamar del Segura por ser los dos observatorios costeros más próximos a la isla. De ambas se han podido obtener en los boletines mensuales del Servicio Meteorológico Nacional, los registros de pluviosidad y temperatura del período comprendido entre 1945 y 1971.

Todos los datos de estos boletines correspondientes a estaciones de la provincia de Alicante y algunos de otras limitrofes, están soportados en un fichero de tarjetas perforadas en el Departamento de Biología y Geología del C.E.U. de Alicante: Para la elaboración de los correspondientes a las estaciones elegidas se aplicó el programa CLIMA escrito en Fortran IV que se procesó en el IBM-370-145 de CETECSA. El programa parte de los datos mensuales de pluviosidad y temperaturas máxima y mínima de los años citados y calcula para cada mes: pluviosidad media, 
TABLA 1. - Datos climatológicos para el periodo 1945-1971 de las estaciones de Alicante (Ciudad Jardín) y Guardamar del Segura.

\section{Estación ALICANTE (Ciudad Jardin)}

Indice de aridez: $12.81 \quad$ Cociente pluviotérmico: 70.73

R

Pluviosidad anual: $359.4 \mathrm{~mm}$.

$\bar{T}_{\text {max }}$

28.6

24.0

19.7

44.6

33.0

21.1

5.0

13.1

43.9

58.9

37.7

29.8
16.0

17.8

20.1

21.9

25.2

27.6

32.0

32.4

29.5

24.8

20.5

17.2

$$
\bar{T}_{\text {min }}
$$

5.9

6.5

8.2

10.0

13.0

15.8

19.3

19.9

17.7

13.6

10.3

7.4
$\bar{T}$

$11 .^{\circ}$

12.1

14.1

15.9

19.1

21.7

25.7

26.1

23.6

19.2

15.4

12.3

Estación GUARDAMAR DEL SEGURA

Indice de aridez: $10.42 \quad$ Cociente Pluviotérmico: 62.38

Pluviosidad anual: $292.1 \mathrm{~mm}$.

\begin{tabular}{|c|c|c|c|c|}
\hline & $\mathbf{R}$ & $\overline{\mathrm{T}}_{\max }$ & $\overline{\mathrm{T}}_{\min }$ & $\overline{\mathrm{T}}$ \\
\hline$E \ldots \ldots \ldots \ldots$ & 25.0 & 15.2 & 6.8 & 11.3 \\
\hline$F \ldots \ldots \ldots$ & 18.8 & 16.8 & 7.2 & 12.0 \\
\hline$\dot{M} \ldots \ldots \ldots \ldots$ & 18.5 & 18.9 & 9.0 & 13.9 \\
\hline$A \ldots \ldots \ldots$ & 27.2 & 21.1 & 10.9 & 16.0 \\
\hline$M \ldots \ldots \ldots$ & 24.4 & 24.3 & 18.7 & 21.5 \\
\hline$J \ldots \ldots \ldots$ & 15.4 & 27.4 & 16.6 & 22.0 \\
\hline$\jmath \ldots \ldots \ldots$ & 4.0 & 30.8 & 19.3 & 25.1 \\
\hline $\mathrm{A} \ldots \ldots \ldots$ & 5.0 & 31.3 & 19.9 & 25.6 \\
\hline$s \ldots \ldots \ldots$ & 29.3 & 28.7 & 17.9 & 23.3 \\
\hline $0 \ldots \ldots \ldots$ & 55.3 & 24.8 & 14.0 & 19.0 \\
\hline$N \ldots \ldots \ldots \ldots$ & 29.4 & 19.2 & 9.7 & 14.5 \\
\hline$D \ldots \ldots \ldots$ & 39.8 & 17.4 & 7.1 & 12.3 \\
\hline
\end{tabular}


temperatura máxima media, temperatura mínima media $y$ temperatura media. Además se obtienen la pluviosidad anual media y las mediąs anuales de máximas, mínimas y medias, junto con el índice de aridez de MARTONNE y el cociente pluviotérmico de EMBERGER. Todos estos resultados obtenidos con el programa CLIMA figuran en la tabla 1. Con ellos se han construido los diagramas climáticos que aparecen en la figura $1, a, b, c$ y $d$, que corresponden a un clima mediterráneo seco.

Según el diagrama de bioclimas mediterráneos de Túnez de BARTOLI y col. (1967) (en GOUNOT, 1969), en el que figuran en ordenadas los cocientes pluviotérmicos de EMBERGER y en abcisas las temperaturas medias del mes más frio, el clima de Alicante sería mediterráneo subhúmedo y el de Guardamar, mediterráneo semiárido. Una diferencia similar se infiere de los índices de aridez de MARTONNE, claramente menor en Guardamar.

Parece lógico considerar el clima de isla Plana más emparentado con el de Guardamar del Segura, y si acaso más extremo aún, que con el de Alicante, Ciudad Jardín. El brazo de mar que separa la isla de la costa es sin duda más semejante al horizonte llano que presenta la parte sur de la provincia de Alicante que a las estribaciones de las sierras alicantinas próximas a la capital.

\subsection{Flora y vegetación}

La flora de Nueva Tabarca está formada por algo menos de un centenar de especies, muchas de ellas plantàs de marcado carácter nitrófilo o nitro-halófilo ya que prácticamente no existe ningún lugar de la superficie insular libre de la influencia antropógena. La flora de Nueva Tabarca no difiere pues de la presente en los alrededores de las poblaciones litorales como Santa Pola o Guardamar, si bien es bastante más pobre en especies.

En la isla se han observado hasta el momento las especies vegetales que figuran en las tabla 2 y que se han dispuesto en seis grupos de abundancia, desde las muy comunes (CCC) a las muy raras (RRR).

La vegetación de la isla de Nueva Tabarca está formada por asociaciones de las clases Rudero-Secalietea, Thero-Brachypodietea y Crithmo-Limonietea, esta última muy poco representada en comparación con las anteriores. 

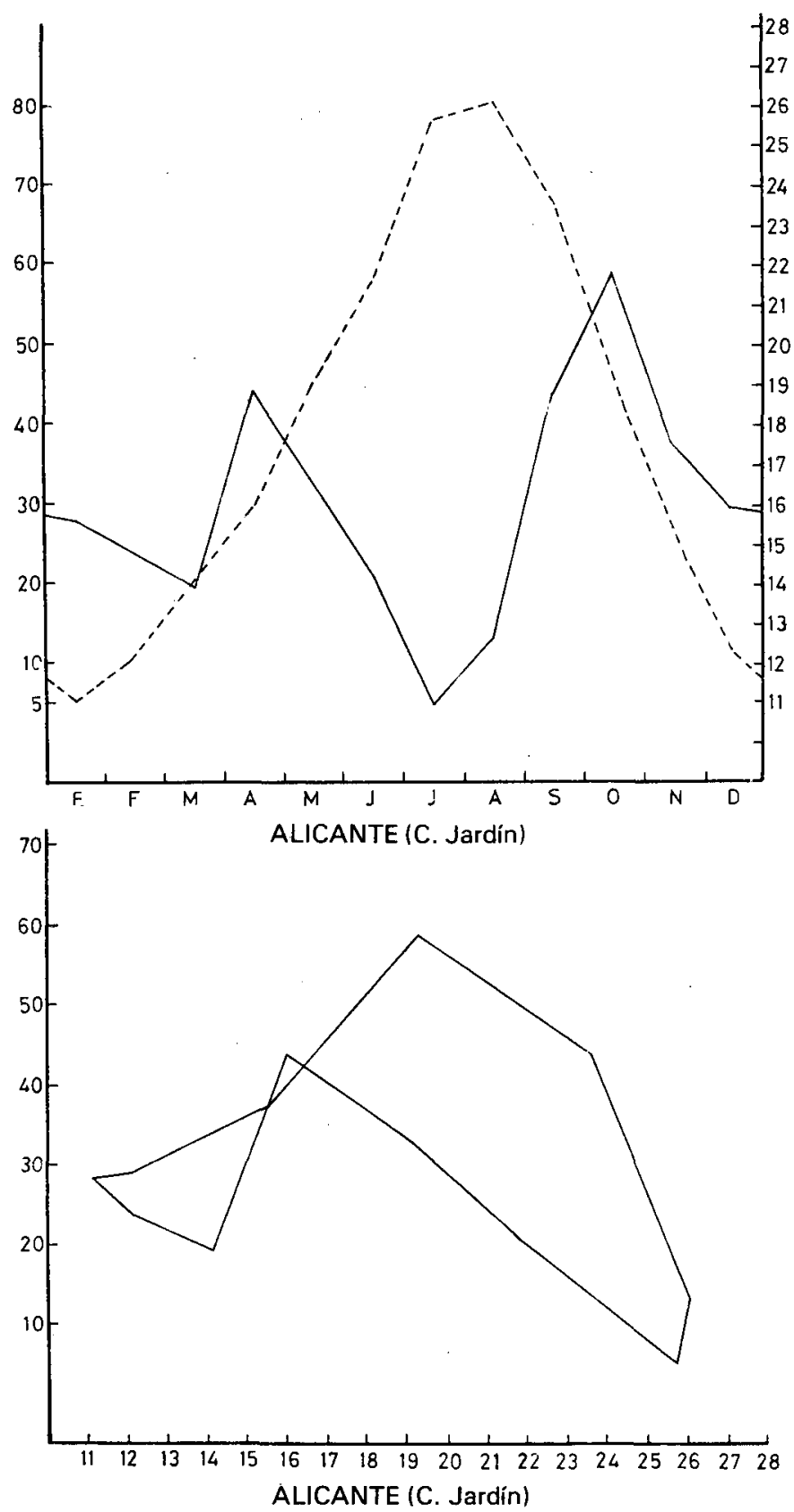

FIGURA 1. - Diagramas ombrotérmicos y climatogramas correspondientes: a y b a la estación de Alicante (C. Jardín); c y d a la estación de Guardamar del Segura. 


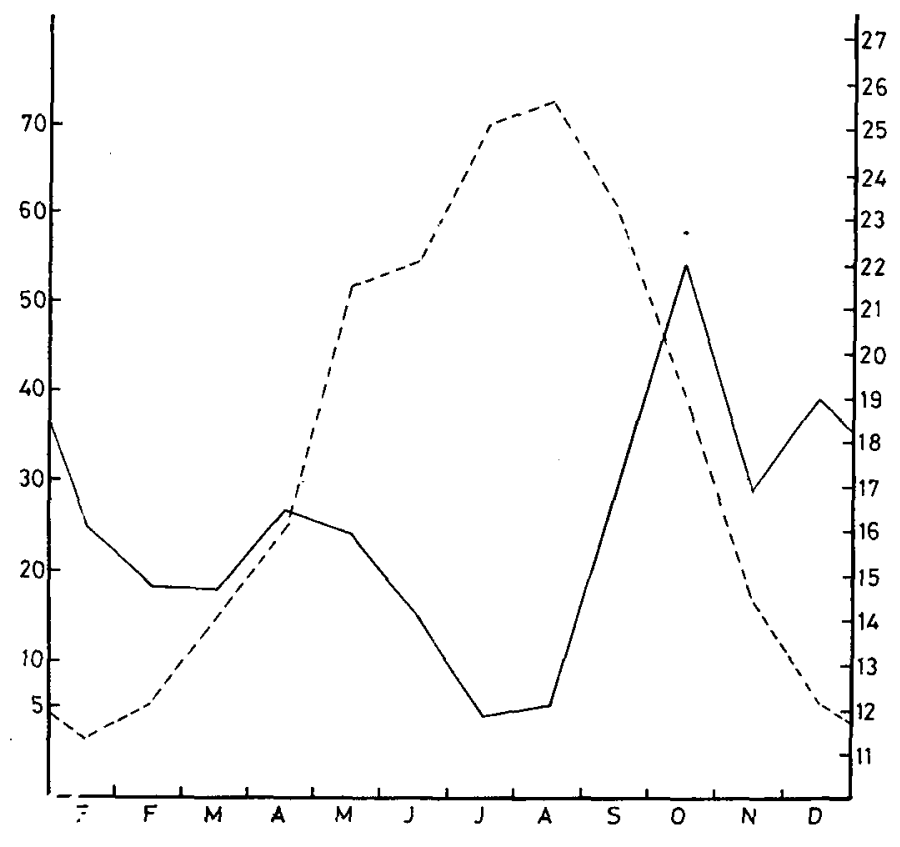

GUARDAMAR DEL SEGURA

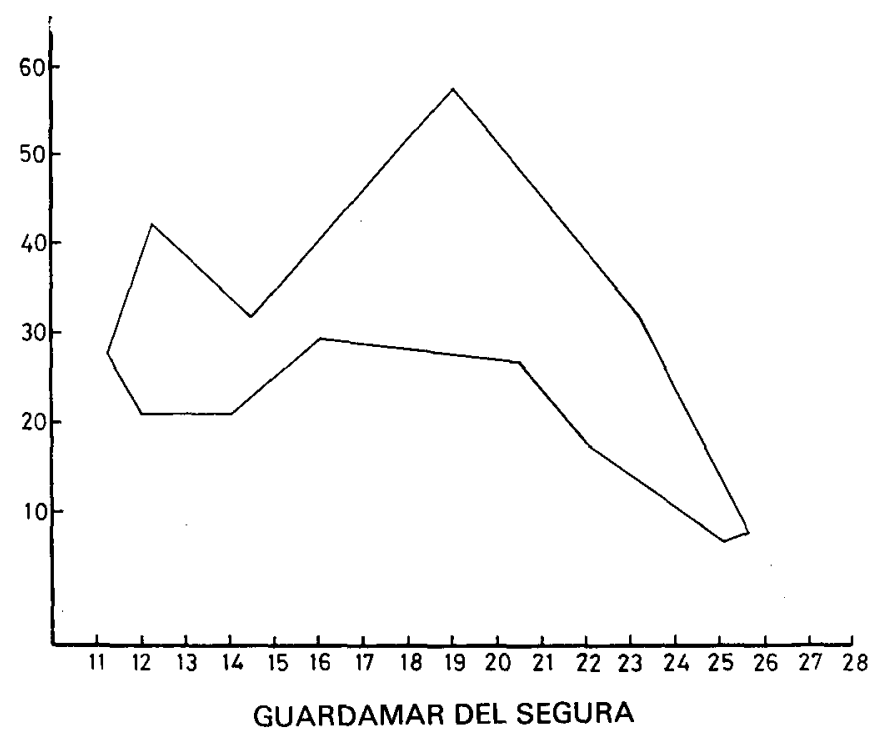


TABLA 2.-Grupos de especies vegetales ordenados por su abundancia, desde CCC (especies muy comunes) hasta RRR (especies muy raras).

CCC) Stipa retorta

Carrichtera annua

Echium vulgare

Mesembryanthemum crystallinum Mesembryanthemum nodiflorum

Suaeda fruticosa ssp. fruticosa

Atriplex glauca

Asteriscus maritimus

Reichardia tingitana ssp. discolor

CC) Launaea nudicaulis

Emex spinosa

Hedypnosis cretica

Convolvulus althaeoides

Medicago littoralis

Euphorbia terracina

Erodium chium

Beta vulgaris ssp. maritima

Malva parviflora

Silene cerastioides

Sonchus tenerrimus

C) Chenopodium murale

Centaurea seridis

Silene apetala

Glaucium flavum

Withania frutescens

Hyoscyamus albus

Lycium intricatum

Pallenis spinosa

Cynodon dactylon

Hordeum murinum ssp. leporinum

Avena sterilis ssp. ludoviciana

Atractylis cancellata

Plantago coronopus

Bupleurum semicompositum

Asphodelus fistulosus

Spergularia diandra

Convolvulus arvensis

Senecio vulgaris

Medicago truncatula

Lavatera mauritanica

Calendula arvensis
R) Urtica urens

Mercurialis annua

Echium parviflorum

Polycarpon tetraphyllon

Galium valantia

Antirrhinum orontium

Cakile maritima

Plantago albicans

Asparagus albus

Filago germanica var. micropodioides

Ajuga iva

Herniaria hirsuta var. cinerea

Brachypodium distachyum

Sisymbrium irio

Crithmum maritimum

Frankenia corymbosa

Alyssum maritimum

Atriplex hastata

Salsola kali

Matthiola parviflora

Lamarckia aurea

RR) Melilotus sulcata

Diplotaxis erucoides

Fumaria sp.

Phagnalon rupestre

Convolvulus siculus

Eruca sativa

Schismus calycinus

Agropyrum junceum

Atriplex cf. rosea

Anagallis arvensis

Urospermum picroides

RRR) Torilis nodosa

Eryngium maritimum

Plantago ovata

Heliotropium curassavicum

Polypogon maritimum

Lygeum spartum

Astragalus $\mathrm{sp}$.

Ononis natrix 
TABLA 3. - Tabla de inventarios sobre superficies de 40-50 metros cuadrados pertenecientes a la alianza Salsolo-peganion.

Caracterísicas territoriales:

Mesembryanthemum nodiflorum .

Mesembryanthemum cristallinum.

Características de la alianza (Salsolo Peganion)

Atriplex glauca.

Características de la clase (Rudero-Secalietea):

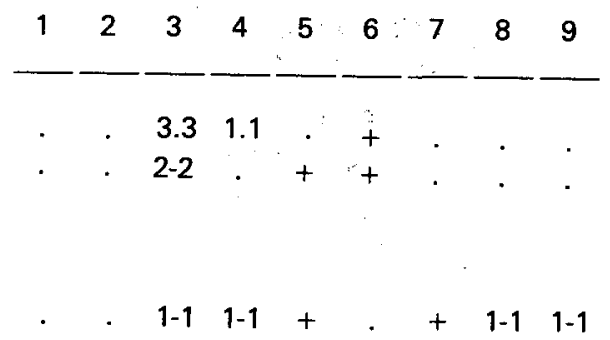

Chenopodium murale

Malva parviflora............ $+2-2,+\quad+2-22-21-1$

Beta vulgaris ssp. maritima ..... . . ..++++++ .

Emex spinosa............ . . . . . . . . $1-1$

Sisymbrium irio ............. . . . . ++++

Hordeum murinum ssp. leporinum.$+++t^{+}$1-1 1-1 +

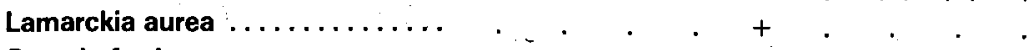

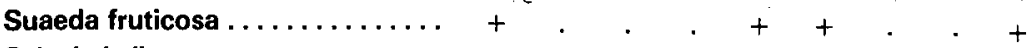

Salsola kali . . . . . . . . . . . . $+\ldots \ldots \ldots .+$

Urtica urens . . . . . . . . . . . . . . 1-1.

Plantago coronopus $\ldots \ldots \ldots \ldots .44-3$ - $3-3+.+$.

Acompañantes:

Reichardia tingitana

Euphorbia terracina

Hyoscyamus albus.

Glaucium flavum

Cakile maritima

Atriplex hastata

Heliotropium curassavicum

Echium vulgare

Acompañantes presentes en un solo inventario:

5: Frankenia corymbosa, Sonchus tenerrimus, Torilis nodosa.

8: Senecio vulgaris.

9: Bupleurum semicompositum. 
En la clase Rudero-Secalietea se incluyen comunidades de ambientes ruderales y tierras de cultivos muy directamente influidos por el hombre. La tabla 3 reúne nueve inventarios tomados en la isla en superficies de 40-50 metros cuadrados, que se consideran pertenecientes a esta clase, y dentro de ella, a la alianza Salsolo-Peganion, que BRAUN-BLANOUET y O. BOLOS $(1954,1957)$ y O. BOLOS (1967) definen como conjunto de comunidades de ambientes nitro-halófilos de la provincia ibero-mauritánica esteparia, que constan de unos pocos caméfitos o nanofanerófitos con un nutrido acompañamiento de pequeños terófitos. La mayoría de los inventarios de la tabla 3 podrían incluirse en la asociación Mesembryanthemetum crystallino-nodiflori descrita por O. BOLOS (1957) y que RIGUAL (1972) incluye en su nueva alianza Salsolo-Suedion comprendida en el orden Atriplicetalia glaucae (RIV.-GODAY).

En Nueva Tabarca, el Salsolo-Peganion se extiende sobre todo por la zona poblada de la parte occidental, pero también se encuentra representado en los alrededores de las demás construcciones de la parte oriental. En la playa, inmediatamente después de los acúmulos de Posidonia oceanica aparece una franja de vegetación con clara dominancia de Cakile maritima en la que se encuentra también Centaurea seridis, Salsola kali, Beta vulgaris ssp. maritima y Atriplex glauca, y que correspondería a un Sporobolo-Centauretum seridis RIVAS GODAY et RIGUAL (1858), asociación del Glaucio-Cakilion TX. 1950 em. nom. O. BOLOS 1962 (=Euphorbion peplis TX. 1950) propia de las playas enriquecidas con materia orgánica.

Gran parte de la porción de la isla que queda al este del puerto estuvo cultivada hasta el año 1960. Según los habitantes de la isla, los cultivos daban buen rendimiento los años de lluvia, y se plantaban sobre todo habas, guisantes, trigo y cebada. Se conserva todavía una plantación de Opuntia de aproximadamente veinte años. Todos estos ex-cultivos han sido invadidos por una vegetación de Thero-Brachypodietea que puede muy bien adscribirse a la alianza Stipion-retortae BR. BL. que comprende comunidades casi exclusivamente formadas por terófitos, propias de la parte maritima del territorio ibero-mauritánico estepario.

En la tabla 4 figuran seis inventarios tomados en superficies de cincuenta metros cuadrados en este tipo de vegetación. En los lugares más pedregosos la colonización parece corresponder a Asteriscus maritimus que BOLOS (1967) da como posible característica de Crithmo-Limonion, pero sugiriendo su mejor representación en Stipion retortae. En otros lugares de tierra más fina la primera fase de colonización la forman las 
TABLA 4: Tabla de inventarios sobre superficies de 50 metros cuadrados pertenecientes a la alianza Stipion-retortae.

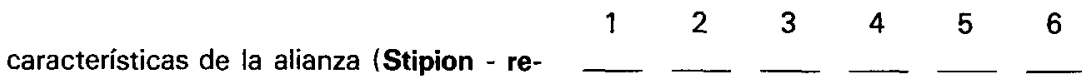
tortae):

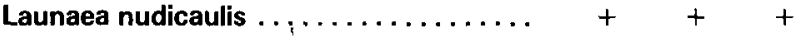

Stipa retorta................. $4-2 \quad 1-1 \quad 3-3 \quad 4$

Reichardia tingitana ssp. discolor ..... $+2-2++++$

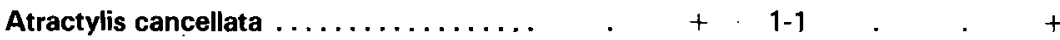

Filago germanica var. micropodioides ... . . . . . . $+{ }_{+}$.

Asteriscus maritimus............. $1-1+3-2++$

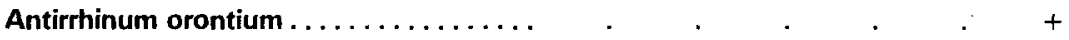

Características del orden (Thero-Brachypodietalia):

Bupleurum semicompositum

Herniaria hirsuta var. cinerea

Características de la clase (Thero-Brachypodietea)

Hedypnosis cretica

Convolvulus althaeoides .............

Medicago littoralis

Acompañantes:

Asphodelus fistulosus

Atriplex glauca

Euphorbia terracina

Beta vulgaris ssp. maritima

Erodium chium

Spergularia diandra

Mesembryanthemum nodiflorum

Emex spinosa

Withania frutescens

Silene cerastioides

Mesembryanthemum crystalinum

Silene apetala. .

Acompañantes presentes en un solo inventario:

1: Echium vulgare, Carrichtera annua.

2: Echium parviflorum, Avena sterilis ssp. Iudoviciana.

3: Convolvulus arvensis, Cynodon dactylon.

4: Sonchus tenerrimus.

5: Lycium intricatum, Polycarpon tetraphyllon, Suaeda fruticosa, Plantago coronopus, Lamarckia aurea, Galium valantia.

6: Malva parviflora, Astragalus sp. 
especies nitrófilas Carrichtera annua y Emex spinosa, si bien es de presumir que en ellos Stipa retorta termina por dominar.

La clase Crithmo-Limonietea está escasamente representada en Nueva Tabarca. El litoral de la parte oriental de la isla consta de una serie de pequeños acantilados de tres o cuatro metros de desnivel que tienen en su base pequeñas playas de grava gruesa y en ellos aparecen matas aisladas de Crithmum maritimum; en la parte occidental, en un roquizal arenoso y con numerosos restos de Posidonia oceanica, el hinojo de mar es bastante más abundante, en una porción de tierra unida a la isla solamente por algunas rocas que afloran del mar es donde mejor representada estaría esta alianza y junto al Crithmum maritimum aparecen Frankenia corymbosa y Plantago coronopus junto a Asteriscus maritimum y Suaeda fruticosa, entre otras especies.

En las cercanías del faro, en los márgenes de un antiguo campo de cultivo llama la atención la existencia de un matorral de hasta $2,5 \mathrm{~m}$. de altura formado por tres vegetales espinosos que presentan una interesante convergencia fisionómica y también fisiológica al ser los tres caducifolios estivales. Estos vegetales son Asparagus albus, Withania frutescens y Lycium intrincatum. Los dos primeros son especies características de la alianza Oleo-Ceratonion y la tercera se da como característica del Salsolo-Peganion aunque se admite (BOLOS 1967) su posible intervención en el Oleo-Ceratonion. Esta maquia espinosa se considera un fragmento del Chamaerops Rhamnetum. O. BOLOS 1950, que hipotéticamente sería la vegetación potencial de la isla.

\subsection{Fauna}

El capítulo de fauna es muy reducido en lo que a vertebrados terrestres se refiere ya que la extensión de la isla es pequeña y las condiciones morfológicas de su superficie no permiten una diversificación del biotopo. Así, los únicos representantes de la clase Mamíferos son la rata (Rattus sp.) que abunda en los terrenos cercanos al pequeño puerto y a dos estercoleros de la parte sur de la isla, el ratón común (Mus musculus) en las zonas más próximas a la población y al menos una especie de quiróptero, probablemente Pipistrellus pipistrellus; como mamíferos domésticos, hay que reseñar un número considerable de gatos y algunos perros que diariamente recorren la isla. Faltan en ella elementos tan extendidos como los lepóridos (ya que el conejo que se introdujo se extinguió hace unos cuantos años) y representantes del orden Insectívoros tan comunes como la musaraña y el erizo. 
Los saurios están representados únicamente por el eslizón ibérico (Chalcides bedriagai) y las dos clases de salamanquesa, la verrugosa (Hemidactylus turcicus), más bien rara, y la común (Tarentola mauritanica) que es bastante más abundante sobre todo en los dos grandes aljibes de la parte central de la isla.

La ausencia total de corrientes de agua dulce y la casi total de recipientes, motivan el que no exista en la isla ninguna especie de anfibio.

En el grupo de los ofidios hay que destacar la presencia de unos pocos ejemplares de culebra bastarda (Malpolon monspessulanus) de las que se han hallado tres mudas de entre 105 y $120 \mathrm{~cm}$.; hace poco se pudo observar en uno de los aljibes, un ejemplar de $150 \mathrm{~cm}$. de longitud total.

Los datos sobre las aves provienen de observaciones directas y de algunas encuestas verbales planteadas a los pobladores. Hay muy pocas especies que aniden en la isla, sin duda por la falta de arbolado. Estrictamente lo hacen: el gorrión (Passer domesticus), en lugares cercanos a la población, en las mismas casas viejas; la totovía (Lullula arborea) y la alondra (Alauda arvensis) en los dos tercios restantes de la isla; un limícolo, posiblemente el correlimos común (Calidris alpina) en los pequeños acantilados; y por otra parte, la golondrina (Hirundo rustica), el avión común (Delinchon urbica) y el vencejo común (Apus apus). El resto de las aves que se observan son visitantes ya que con facilidad atraviesan los pocos kilómetros que separan la isla del cabo de Santa Pola, y son las mismas que forman la avifauna litoral. Incluso las dos especies de gaviotas (Larus argentatus y Larus ridibundus) prefieren salvo alguna excepción, anidar en las costas del cabo. Junto a ellas puede verse en los meses de septiembre-octubre algún charrancito (Sterna albifrons) o fumarel común (Chlidonis niger) asi como alguna bandada de patos.

La abubilla (Upupa epops) es un ave común de la isla y, procedente al parecer de la pequeña meseta superior del cabo, hace su aparición gran parte de los días. En su tiempo se dejan ver igualmente el mirlo (Turdus merula) y el zorzal charlo (Turdus philomelos).

En los meses de abril y mayo suelen llegar, procedentes de las costas cercanas, algunos ejemplares de tórtola (Streptopelia turtur). No es difícil ver en Nueva Tabarca en estos mismos meses ejemplares de collalba gris (Oenanthe oenanthe), collalba negra (Oenanthe leucura), colirrojo tizón (Phoenicurus ochruros), petirrojo (Erithacus rubecula), ruiseñor común (Lucinia megarhynchos), curruca sarda (Sy/via sarda), lavan- 
dera blanca (Motacilla alba), lavandera boyera (Motacilla flava) y alcaudón común (Lanius senator).

Mención aparte merecen el verdecillo (Serinus serinus), el verderón común (Carduelis chloris) y el jilguero (Carduelis carduelis) que son los representantes con mayor número de individuos después de los alaudidos y golondrinas aunque no aniden en la isla.

De rapaces, esporádicamente suele verse algún halcón (Falco peregrinus) procedente de los roquedos del cabo de Santa Pola; y la lechuza común (Tyto alba) que era elemento permanente en las bóvedas de la iglesia hasta hace unos cuantos años en que desapareció totalmente.

Los datos de la fauna entomológica están reforzados por un trabajo de F. ESPAÑOL (1965) sobre Nueva Tabarca. Es característica de la isla la pobreza en especies, quizás por las condiciones climáticas a las que están sometidas así como al llano perfil y ausencia de agua. Carece de asociaciones halófilas y sabulicolas por no tener terrenos parcialmente inundados ni dunas, típicos del litoral de Santa Pola. Sin embargo, están muy desarrolladas las asociaciones lapidícolas y fitófilas, atraídas por determinadas quenopodiáceas (Atriplex y Suaeda). Ocurre así que ciertos grupos encuentran un hábitat idóneo y abundan excesivamente.

Los grupos y especies más representativas son: Dermápteros (Forficula auricularia), Heterópteros (Dicyphus bolivari, Nysius ericae, Sciocoris carayoni, Geotomus punctulatus), Coleópteros (Tentyria laevis, Morica hybrida, Akis acuminata, Scaurus punctatus, Pimelia baetica, Blaps gigas, Blaps sulcata subsp. brachyura, Micrositus obesus, Micrositus gibbulus, Bulaea lichatschovi, Coccinella 7-punctata, Timarcha intermedia, Oxylepis deflexicollis, Phytonomus variabilis, Orthomus barbarus, Licinus punctatulus, Masoreus wetterhalli, Cymindis lineola).

Dentro de la microfauna, merecen especial atención ciertos moluscos cuyo mejor representante es Theba pisana, que invade completamente la isla; con la misma frecuencia lo hacen algunos isópodos (Porcellio sp.).

Entre la población de arácnidos, destaca la desproporcionada abundancia de licósidos ( $L y c o s a$ tarentula) que ocupa prácticamente los dos tercios aplanados de la isla; por lo demás, ejemplares comunes a excepción del grupo de argiópidos representados por Argiope sp. 


\subsection{Taxonomia}

\subsubsection{Sistemática del Eslizón ibérico}

Al describir BOSCA (1880) la subespecie Gongilus ocellatus bedriagai hizo hincapié en las acusadas diferencias que lo separaban de los ejemplares africanos de Argelia dados como Gongilus ocellatus tipo. Las características diferenciales propuestas son: la pequeñez de las patas a las que dice poder aplicárseles el calificativo de rudimentarias, ya que las anteriores extendidas sólo alcanzan el orificio auricular y las posteriores son como una tercera parte de la longitud del cuerpo, en la hembra; en la parte dorsal de los dedos de las patas anteriores y posteriores hay una escama menos, y también hay una vértebra lumbar menos; el tamaño es mucho menor, con una longitud total media de unos $125 \mathrm{~mm}$.

Con respecto al modelo de coloración habla BOSCA de una zona media de color castaño a lo largo del dorso con una semizona más clara a cada costado. Estas franjas dorsales van desde el borde posterior de los escudos cefálicos hasta la mitad de la cola y en ellas hay un número variable de manchas oceliformes. Admite el citado autor la existencia de distintos tipos de dibujos y coloración y de transiciones insensibles entre ellos.

Las descripciones más detalladas las hace BOSCA sobre dos ejemplares hembras de Dosaguas (Valencia), si bien habla de otros semejantes de Luchente (Valencia) y de otra variedad de La Caracollera (Ciudad Real).

BOULENGUER (1887) eleva la subespecie de BOSCA a la categoria de especie con el nombre de Chalcides bedriagai y MERTENS y MULLER dan a Dosaguas, provincia de Valencia, como localidad típica de la especie.

En las claves dicotómicas de su obra, MERTENS ( 1960) utiliza para la identificación de Chalcides bedriagai unos caracteres que resultan bastante en desacuerdo con muchos ejemplares de la península. Para este autor, la placa loreal cabalga únicamente sobre la segunda escama supralabial y el número de filas de escamas alrededor del cuerpo es de 26-28. DOTTRENS (1963) repite el carácter diferencial de la placa loreal 


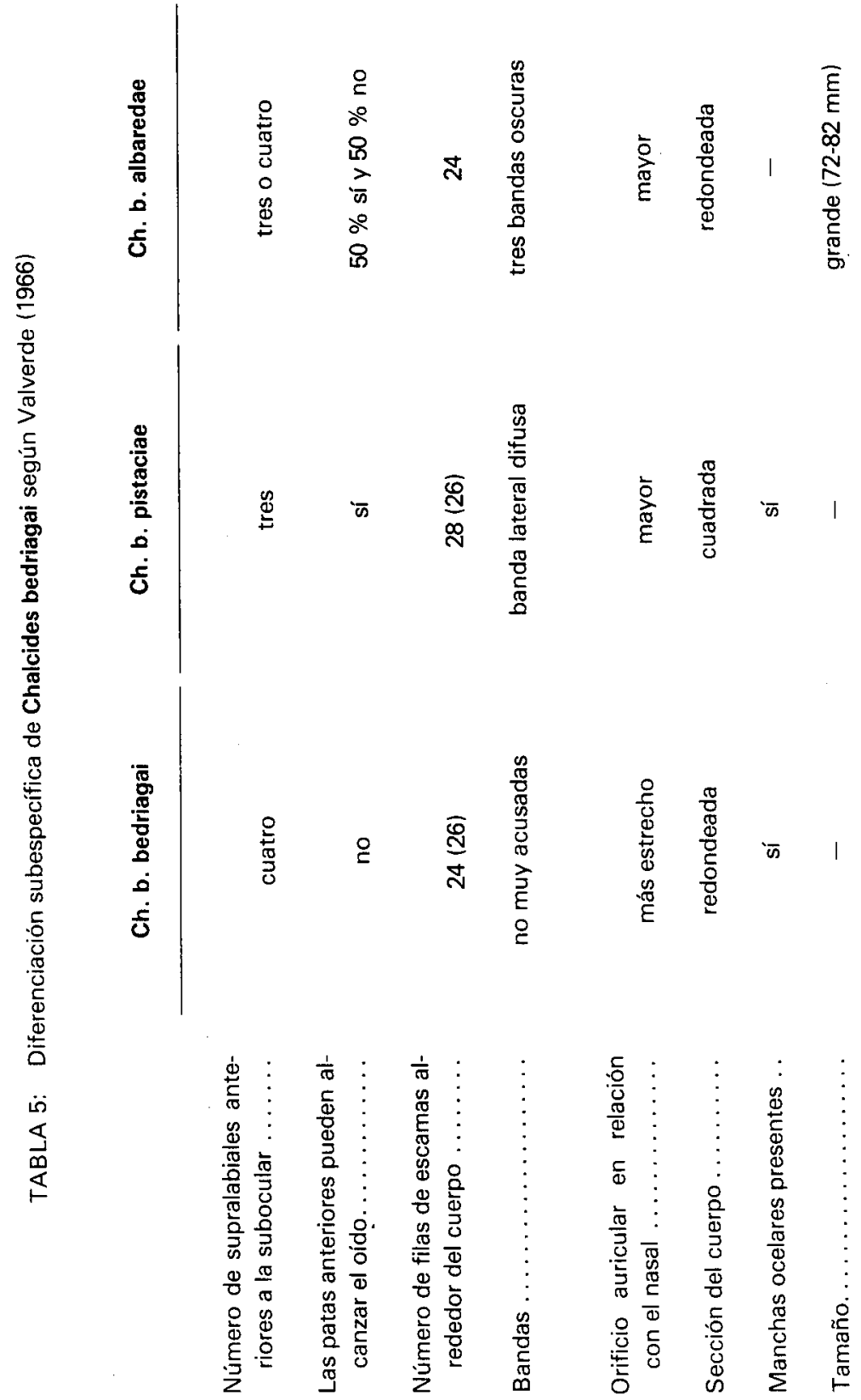




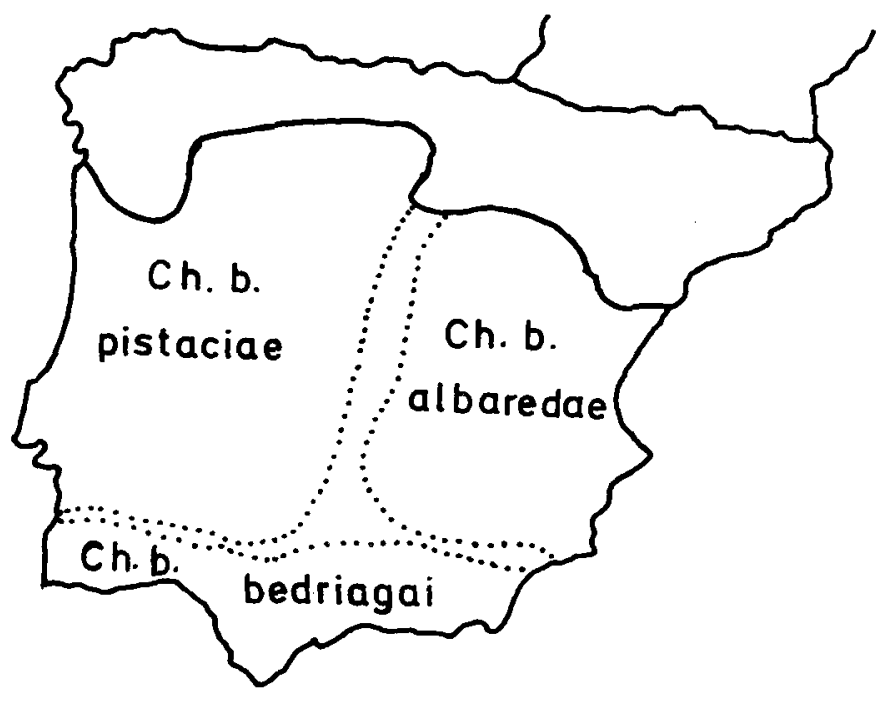

FIGURA 2. - Interpretación aproximada de la distribución de las tres subespecies de eslizón ibérico.

y es todavía menos afortunado al destacar como carácter diferencial de la especie frente a Chalcides ocellatus la ausencia de manchas oceliformes.

VALVERDE (1966) realiza una revisión de los Chalcides bedriagai de diversas localidades de la península incluyendo Portugal, y descubre unas diferencias que lo llevan al establecimiento de tres nuevas subespecies: Chalcides bedriagai bedriagai, Chalcides bedriagai pistaciae y Chalcides bedriagai albaredae, localizada la primera en Murcia, Almería y toda la costa hasta Huelva; en España occidental y Portugal la segunda; y en las montañas del este de España, Teruel, Tarragona y Castellón, la tercera (Fig. 2). Los caracteres en que VALVERDE basa su diferenciación subespecífica vienen dados en la tabla 5 . El mencionado autor sugiere que BOSCA estudió ejemplares intermedios entre Chalcides bedriagai bedriagai y Chalcides bedriagai albaredae de la provincia de Valencia (Dosaguas y Luchente) y ejemplares de Chalcides bedriagai pistaciae de La Caracollera (Ciudad Real), y al no haber designado BOSCA ejemplar tipo, considera como típica la subespecie que más difiere de Chalcides ocellatus, pero, tal como indica recientemente SALVADOR (1974) olvida que ya se había designado localidad típica. 


\subsubsection{Definición de caracteres}

Son dieciocho los caracteres que se han utilizado en este estudio, once de los cuales son cuantitativos (diez continuos y uno merístico), y los restantes seis, binarios o multiestados. Algunos de estos caracteres son los que aparecen en las descripciones que hacen BOSCA (1880) y VALVERDE (1966), mientras que otros son nuevos. La descripción de los caracteres es la que sigue:

Cuantitativos:

Carácter 1: Longitud de la cabeza y del cuerpo (LCC).

Carácter 2: Número de filas de escamas alrededor del cuerpo.

Carácter 3: Distancia entre patas anteriores y posteriores.

Carácter 4: Longitud de las extremidades anteriores. Carácter ambiguo por lo difícil que resulta precisar la situación exacta del comienzo del brazo.

Carácter 5: Longitud de las extremidades posteriores. Medido al igual que el anterior y con las mismas dificultades.

Carácter 6: Distancia desde el orificio nasal al auditivo.

Carácter 7. Distancia entre el extremo del hocico y el borde de las últimas placas cefálicas.

Carácter 8: Distancia entre el orificio auditivo y el arranque del brazo.

Carácter 9: Anchura de la franja central oscura. Medido en la zona de la nuca.

Carácter 10: Anchura de la placa frontal. Carácter cuantitativo continuo medido en las unidades de un ocular graduado. Equivalencia: $1 \mathrm{mi}-$ limetro $=13$ unidades.

Carácter 11: Longitud de la placa frontal. Carácter cuantitativo continuo medido en las mismas unidades y condiciones que el carácter anterior. 


\section{Binarios y multiestados:}

Carácter 1: Sexo. Carácter binario. La diferenciación se realiza por diformismo en el aparato gonadal, correspondiendo el estado 1 al macho y el 2 a la hembra.

Carácter 2: Número de escamas supralabiales. Son dos las medidas y cuatro los estados: mediante una visión dorsal del animal, la primera es la del lado izquierdo, y la segunda, la del derecho. En cada uno, puede tener tres o cuatro de estas escamas anteriores a la subocular, $y$ las combinaciones que resultan son las siguientes: $3-3(1), 3-4(2), 4-3$ (3), $4-4$ y 5-5 (4).

Carácter 3: Las patas anteriores pueden alcanzar el oído. Las patas delanteras alargadas hacia delante, llegan, no lo hacen o sobrepasan el orificio auditivo. Carácter multiestado con tres estados: llegan (1), no llegan (2) o sobrepasan (3).

Carácter 4: Posición de la escama loreal a ambos lados de la cabeza. La base de esta escama puede descansar sobre una escama supralabial o bien estar compartida entre dos de ellas. Carácter con cuatro estados y dos medidas como el carácter número 2: $2 / 2(1), 2 / 2-3(2), 2-3 / 2$ (3), $2-3 / 2-3(4)$.

Carácter 5: Disposición de los ocelos a lo largo del cuerpo. Carácter con cuatro estados: ocelos en la región sacra y principio de la cola (1), ocelos en esta misma región y en la de la nuca (2), ocelos sobre todo el dorso (3), y sin ocelos (0).

Carácter 6: Bandas. Tres rayas oscuras longitudinales bien marcadas en el dorso; una en el centro y otra a cada lado, separadas de la central por dos más pálidas. Carácter binario: con tres rayas (1) y $\sin$ ellas (2).

Carácter 7: Número de escamas infralabiales. Las escamas están contadas hasta el nivel de la posterior a la subocular sin sobrepasarla. Carácter con cuatro estados al igual que el carácter número dos, así como las dos posiciones correspondientes a los dos lados de la cabeza. En cada una de las posiciones encontramos cuatro o cinco de estas escamas siendo las combinaciones y los estados: 4-4 (1), 4-5 (2), 5-4 (3), 5-5 (4). 
TABLA 6: Valores de los once caracteres numéricos en la totalidad de la muestra de eslizones de N. Tabarca.

\begin{tabular}{|c|c|c|c|c|}
\hline $\begin{array}{l}\text { Caracteres } \\
\text { numéricos }\end{array}$ & $\begin{array}{l}\text { Valor } \\
\text { minimo }\end{array}$ & $\begin{array}{c}\text { Valor } \\
\text { máximo }\end{array}$ & Medias & $\begin{array}{l}\text { Desv. } \\
\text { típicas }\end{array}$ \\
\hline 1 & 28 & 74 & 56.91 & 11.94 \\
\hline 2 & 24 & 27 & 25.11 & 0.74 \\
\hline 3 & 17.5 & 55 & 38.95 & 9.41 \\
\hline 4 & 5.5 & 10.0 & 7.84 & 1.43 \\
\hline 5 & 7.6 & 16.0 & 12.75 & 2.02 \\
\hline 6 & 5.2 & 9.3 & 7.59 & 1.06 \\
\hline 7 & 5.0 & 9.3 & 7.24 & 0.98 \\
\hline 8 & 4.1 & 9.9 & 7.15 & 1.28 \\
\hline 9 & 1.7 & 4.7 & 2.34 & 1.09 \\
\hline 10 & 24 & 40 & 33.07 & 4.13 \\
\hline 11 & 29 & 52 & 43.19 & 5.38 \\
\hline
\end{tabular}


TABLA 7: Porcentaje de cada uno de los estados de los siete caracteres multiestado en la muestra de eslizones de $\mathrm{N}$. Tabarca.

\section{CARACTERES MULTIESTADO}

\section{Carácter 1}

Indiferenciados........... $\quad 16 \%$

Machos............................. $39 \%$

Hembras

\section{Carácter 3}

Alcanzan ............. $53.3 \%$

No alcanzan ........... $22.3 \%$

Sobrepasan........... $24.3 \%$

\section{Carácter 5}

Sin ocelos............. $4 \%$

Región sacra y cola ......... $14 \%$

Región sacra y cuello ...... . $3 \%$

Todo el cuerpo ocelado .... . $79 \%$

\section{Carăcter 2}

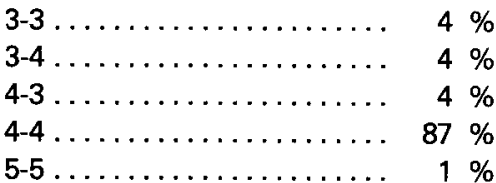

\section{Carácter 4}

$2 / 2 \ldots \ldots \ldots \ldots \ldots \ldots \ldots .5 \%$

$2 / 2-3 \ldots \ldots \ldots \ldots \ldots \ldots . \quad 3 \%$

$2-3 / 2 \ldots \ldots \ldots \ldots \ldots \ldots . \quad 4 \%$

$2-3 / 2-3 \ldots \ldots \ldots \ldots \ldots \ldots \quad 88 \%$

\section{Carácter 6}

Con tres franjas .......... $87 \%$

Sin tres franjas........... $13 \%$

\section{Carácter 7}

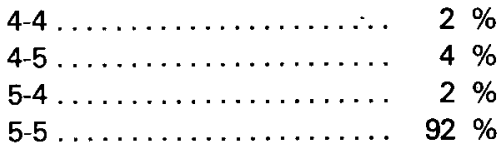




\subsubsection{Ejemplares estudiados}

El número total de ejemplares de eslizón ibérico recogidos en Nueva Tabarca es de cien. La mayor parte de la captura se realizó en dos excursiones a la isla en el mes de julio de 1972 con noventa y un individuos, y en una tercera excursión de captura en abril de 1974 se completó esta cantidad hasta cien. El motivo de esta segunda captura era el de recoger ejemplares en celo con gónadas maduras para la interpretación exacta de la morfología del sexo. Todos estos especímenes se hallan etiquetados en el laboratorio de Biología del C.E.U. de Alicante.

En todos ellos se han apreciado la totalidad de los caracteres que se detailaron en el apartado anterior. Con los datos de los caracteres cuantitativos continuos y meristicos, se han elaborado once histogramas representados en las figuras 3 y 4 . Asimismo se han hallado el valor mínimo, el valor máximo, la media y la desviación típica correspondientes a cada uno de estos once caracteres numéricos y que se detallan en la tabla 6.

Con los datos de los siete caracteres multiestado se han hallado los porcentajes que corresponden a cada estado en cada uno de los caracteres (tabla 7).

Un segundo grupo de datos pertenecen a catorce ejemplares medidos en el Museo de Ciencias Naturales de Madrid, de la colección de Boscá, a tres del laboratorio de la Estación Biológica de Doñana, a diez del Centro de Biología Experimental de Jaca (Huesca) y a seis ejemplares de distintos puntos de la provincia de Alicante, no recogidos en la isla de Nueva Tabarca.

Dos de los individuos medidos en Madrid proceden de Valencia, cinco de la isla de Nueva Tabarca, uno de El Escorial, uno de Málaga y cinco del Mar Menor.

De los tres ejemplares de Sevilla, se desconoce la procedencia.

De los diez individuos de Jaca, cinco pertenecen a Castellón, tres a Tortosa (Tarragona), uno a Nueva Tabarca y uno a Almería.

Los seis ejemplares de la provincia de Alicante, son de Biar, Torrevieja, Sierra de Maigmó, Playa de Mascarat y Monte Benacantil. 

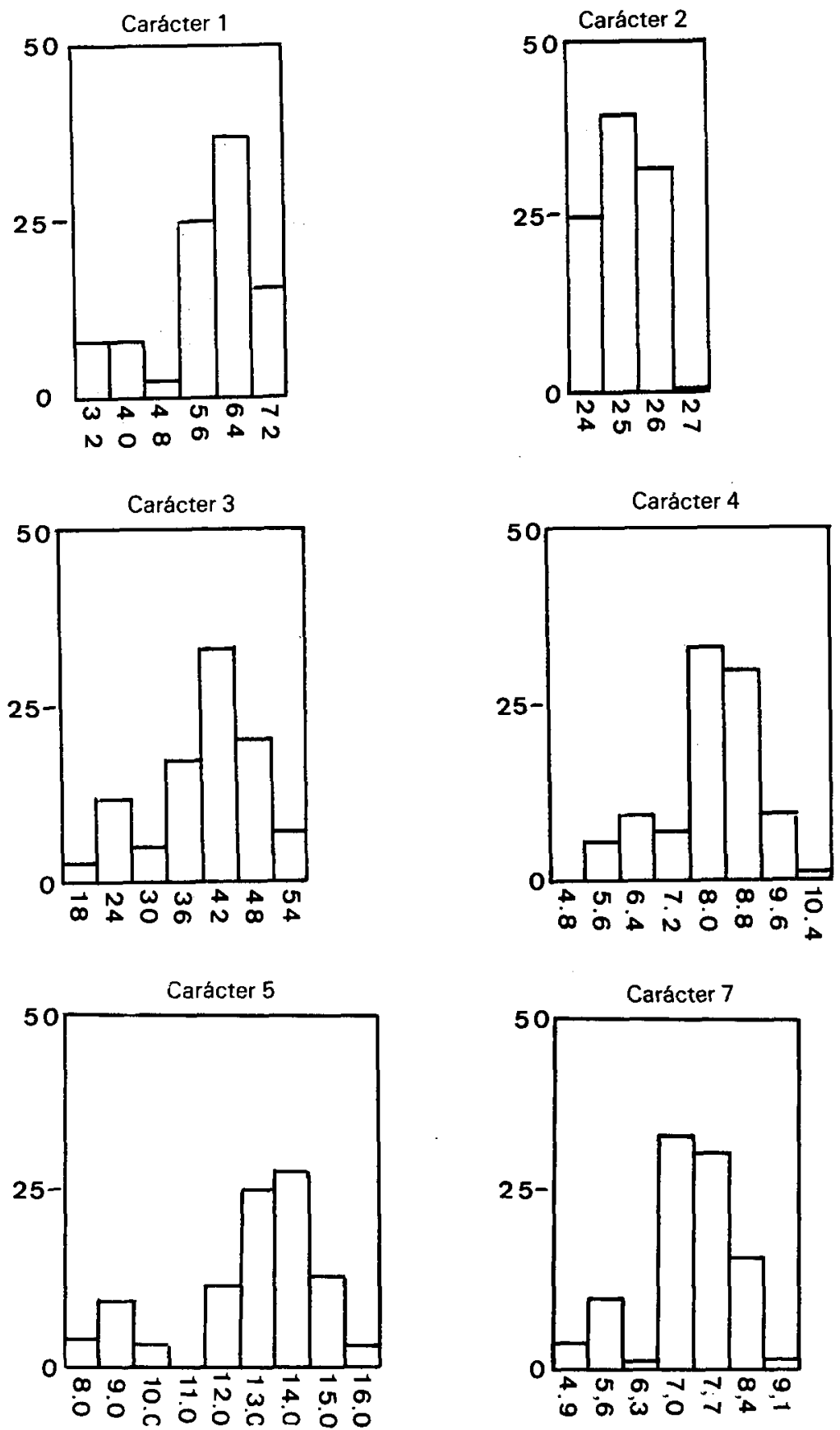

FIGURA 4. - Histogramas elaborados a partir de los datos de los caracteres cuantitativos en la muestra de eslizones de N. Tabarca. 

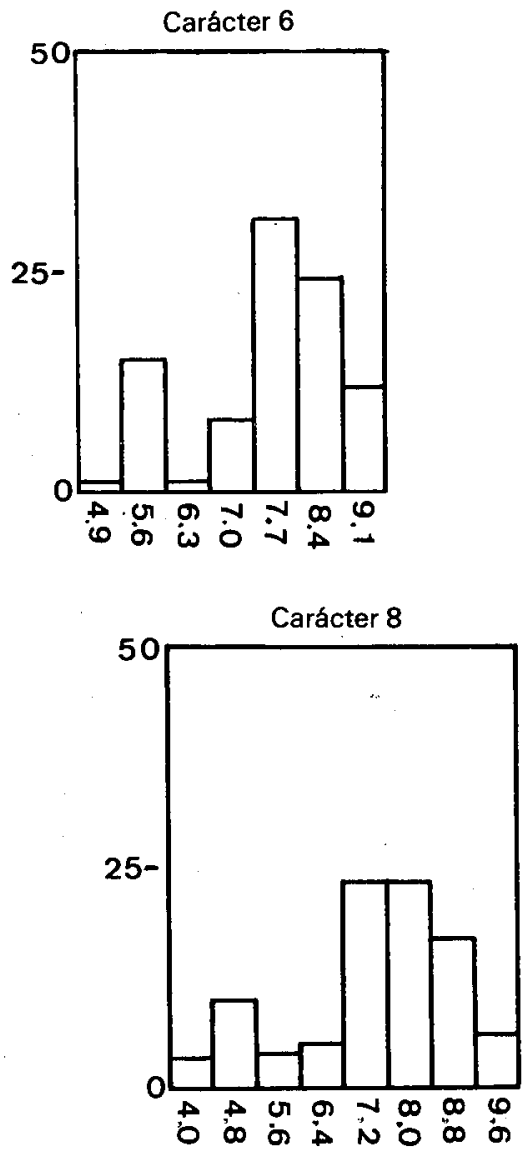
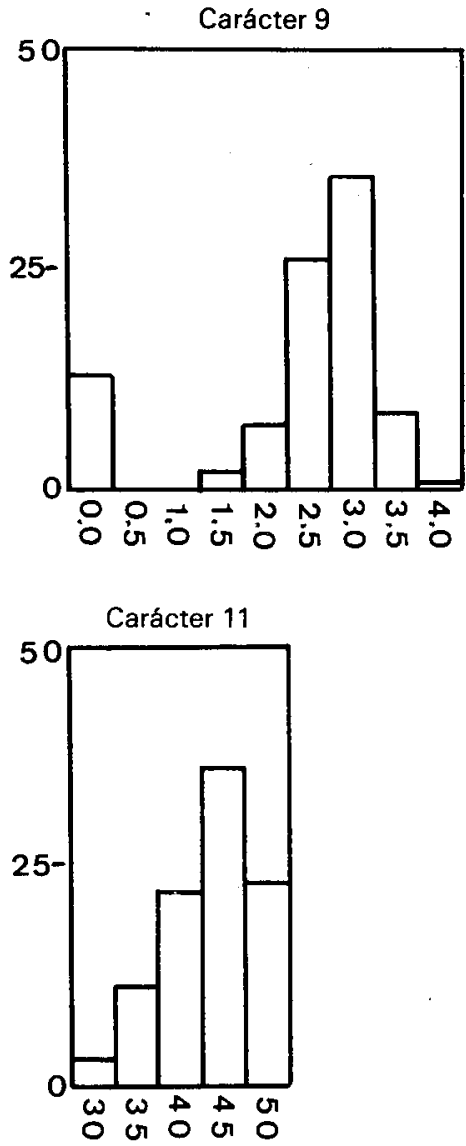

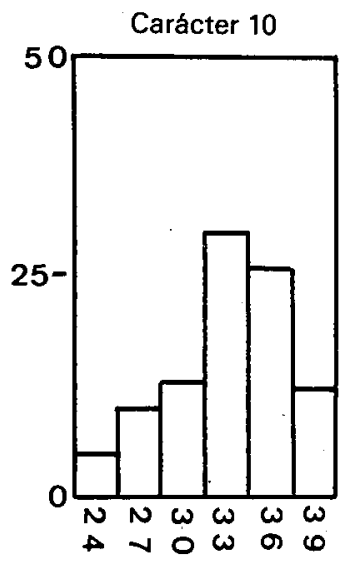

FIGURA 3. - Histogramas elaborados a partir de los datos de los caracteres cuantitativos en la muestra de eslizones de N. Tabarca. 


\subsubsection{Modelo de correlación de los caracteres.}

La investigación de la correlación de caracteres se ha abordado con dos metodologías distintas, la una sólo apta para los atributos numéricos y la otra limitada a caracteres multiestados. La primera de ellas es el análisis factorial, y la segunda, el llamado análisis de caracteres.

El análisis factorial se ha definido de forma general (GOUNOT, 1969) como un método de estudio de la estructura interna de las matrices de varianza y correlación, y de manera más descriptiva, por CUADRAS (1972) como "una técnica estadístico-matemática encaminada a reducir la dimensión de un conjunto de $n$ variables aleatorias observables, expresándolas según un modelo lineal, en función de un número menor de variables hipotéticas llamadas factores comunes».

De las técnicas de análisis factorial, la más comúnmente empleada es el análisis de componentes principales que puede considerarse como un caso particular del modelo factorial más general descrito por HARMAN (1967) en el que las variables de partida se estimarían sólo por los factores comunes (comunalidades) y se prescindiría de los factores únicos (unicidades), que son los que representan la variabilidad no explicada por las comunalidades.

Existe ya una extensa bibliografía sobre la aplicación a problemas taxonómicos de éstas y otras técnicas denominadas en conjunto métodos de ordenación, buena parte de la cual está recopilada en SNEATH y SOKAL (1973).

La aplicación del análisis factorial a los datos de la población de eslizón ibérico de Nueva Tabarca, se hizo por medio del programa FACTO de CUADRAS (1972) que calcula las medias, desviaciones típicas y matriz de correlaciones, los valores propios y el número de factores comunes, el porcentaje acumulativo de los valores propios, los vectores propios y la matriz factorial antes y después de una rotación ortogonal varimax, y las medias en factores comunes; tiene además la opción para el cálculo de una rotación oblicua promax.

El programa FACTO se compiló y ejecutó en IBM 370-145 de CETECSA con 81 de los individuos de la primera recolección. Los resultados obtenidos son poco discriminatorios del valor de los caracteres ya 
que un único factor domina el conjunto de las once variables numéricas consideradas, cuyo espacio teórico, de tantas dimensiones como variables en el caso de que no existiera correlación ninguna entre ellas, se reduce en realidad a una sola dimensión ya que todas representan una única característica: el tamaño.

En la tabla 8 figura la matriz de correlaciones y en la 9 los resultados obtenidos con el método del factor principal.

Con la simple inspección de la matriz de correlaciones resalta ya que, salvo el carácter merístico del número de escamas del contorno, que presenta correlaciones muy bajas con los restantes, todos los demás forman un único grupo de correlación. De acuerdo con ello, el autovalor del primero y único factor extraído, representa el 78,48 por ciento de la variabilidad total.

La redundancia de las variables usadas se aprecia muy clarameniə en sus autovectores, que en nueve de ellos están comprendidos entre -0.31 y -0.33 ; el vector propio del carácter longitud de las patas anteriores es algo mayor $(-0.23)$ y el máximo le corresponde al número de escamas del contorno $(-0.04)$.

El segundo de los métodos empleados es el análisis de caracteres de LEGENDRE y ROGERS (1972) que presentan el programa CHARANAL escrito en Fortran IV (FLEMING y APPAN, 1971). Se ha utilizado una versión original en Fortran IV realizada en el Departamento de Informática del C.E.U. de Alicante y-que consta de un programa principal (ANACAR) y de una subrutina (INFOR). El programa se ha compilado y ejecutado desde la terminal Uniscope 100 del C.E.U. de Alicante conectada al ordenador Univac 1108 del Centro de Proceso de Datos del Ministerio de Educación y Ciencia de Madrid.

Los aspectos teóricos del análisis de los caracteres están tratados en ESTABROOK (1967) y LEGENDRE y ROGERS (1972). Se trata de un modelo basado en la teoría de la información aplicable a caracteres multiestados, en los que, lógicamente, quedan incluidos los binariọs.

A cada carácter con varios estados le corresponde una distribución de probabilidad para los mismos en la serie de individuos considerados, y para ella se puede calcular una entropía incondicional según la expresión de SHANNON (1948)

$$
\mathrm{H}=-\sum_{\mathrm{i}=\mathrm{i}}^{\mathrm{i}=\mathrm{n}} \mathrm{Pi} \log _{2} \mathrm{Pi}
$$

que representa una medida de la incertidumbre con que se puede prever el estado que le corresponderá a un cierto ejemplar. 
TABLA 8: Matriz de los coeficientes de correlación entre los once atributos numéricos.

COEFICIENTES DE CORRELACION

Caracteres

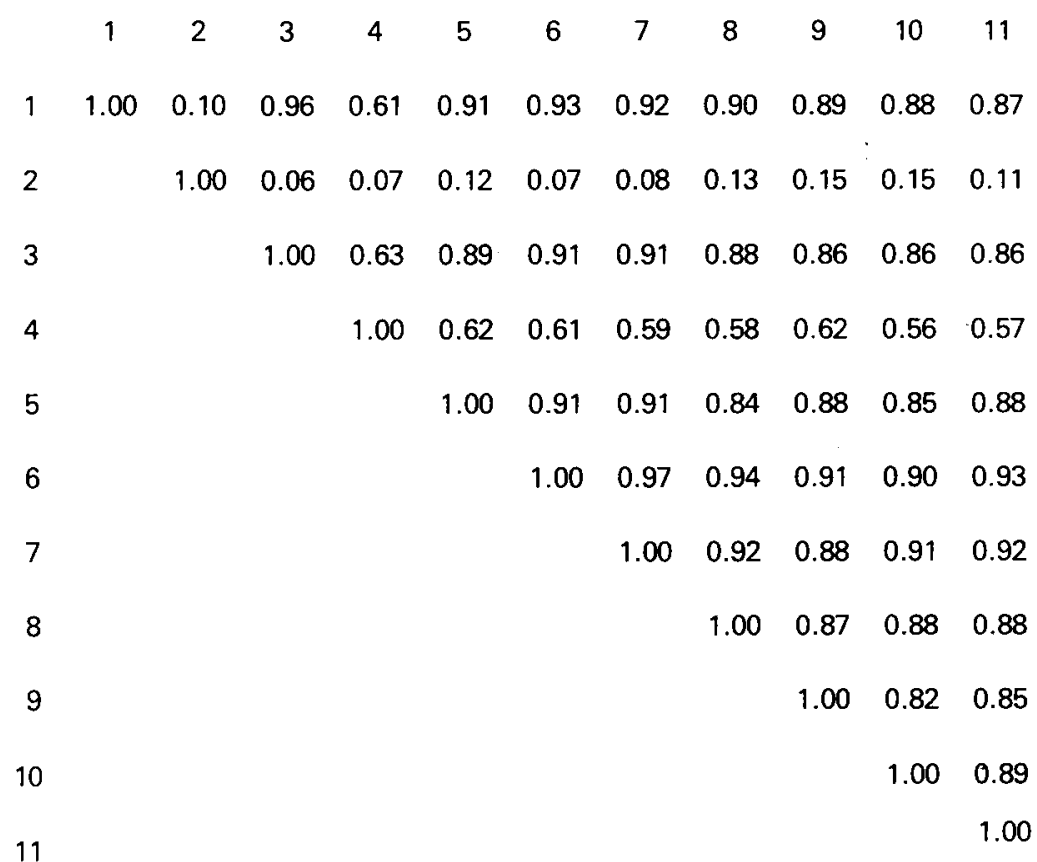

Para cada pareja de caracteres se construye una matriz de probabilidades condicionales: para cada estado de uno de los caracteres, se calculan las probabilidades de cada uno de los estados del otro. El primer atributo representa las filas (i) y el segundo las columnas ( $\mathrm{J}$ ) de la matriz de probabilidades condicionales. Para cada fila de la matriz se calcula una entropía que se multiplica por la probabilidad del estado del carácter a que corresponde la fila. La suma de los productos correspondientes a todos los estados del carácter se define como la entropía condicional que permanece en el estado $J$ después de haber observado el I. 
TABLA 9. - Autovalor del primer eje obtenido por el método del factor principal y los autovectores correspondierites a los once atributos numéricos.

Autovalor $=8.63353$

Porcentaje acumulativo de autovalores $=0.78487$

\begin{tabular}{|c|c|c|c|}
\hline & & Autovectores & Vector factor \\
\hline Carácter & $1 \ldots \ldots \ldots \ldots$ & -0.32828 & -0.96380 \\
\hline Carácter & $2 \ldots \ldots \ldots \ldots$ & -0.04547 & -0.11914 \\
\hline Carácter & 3. & -0.32369 & -0.94667 \\
\hline Carácter & $4 \ldots \ldots \ldots \ldots$ & -0.23078 & -0.63603 \\
\hline Carácter & $5 \ldots \ldots \ldots \ldots$ & -0.32187 & -0.93982 \\
\hline Carácter & $6 \ldots \ldots \ldots \ldots$ & -0.33334 & -0.98307 \\
\hline Carácter & $7 \ldots \ldots \ldots \ldots$ & -0.33054 & -0.97255 \\
\hline Carácter & $8 \ldots \ldots \ldots \ldots$ & -0.32176 & -0.93965 \\
\hline Carácter & 9. & -0.31784 & -0.92491 \\
\hline Carácter & $10 \ldots \ldots \ldots \ldots$ & -0.31706 & -0.92243 \\
\hline Carácter & $11 \ldots \ldots \ldots \ldots$ & -0.32107 & -0.93722 \\
\hline
\end{tabular}

La entropía incondicional de un carácter menos la entropía condicional que permanece en él, después de observar el otro, es una medida de la información que comparten ambos caracteres.

Se puede definir entonces una medida de la independencia de dos caracteres como el cociente de la suma de sus respectivas entropías 
condicionales, partida por la información total de ambos que es lógicamente lo mismo que figura en el numerador más la información que poseen en común. La distancia así definida $D(l, J)$ se puede utilizar directamente o bien se recurre a la similaridad $S(I, J)$ definida como $S(I, J)$ $=1-\mathrm{D}(\mathrm{l}, \mathrm{J})$.

Para cada carácter se calculan dos sumas de las fracciones de la información común con cada otro atributo: la suma de cocientes entre la información compartida y la entropía incondicional de cada uno de los caracteres comparados (SUMRAT) y la suma de cocientes entre los mismos numeradores y la entropia incondicional del propio carácter (SAMRAT).

En la interpretación de resultados hay que tener presente que valores de $S$ mayores de 0.5 representan características muy altamente correlacionadas; valores entre 0.5 y 0.3 corresponden a correlaciones altas $y$ entre 0.1 y 0.3 indican la existencia de correlación. Los valores de SUMRAT y SAMRAT sirven para indicar qué caracteres son los que resumen mayor cantidad de la información de los demás.

En el presente análisis en el que todas las variables se manejan como multiestados en los caracteres utilizados, les corresponde la siguiente numeración:

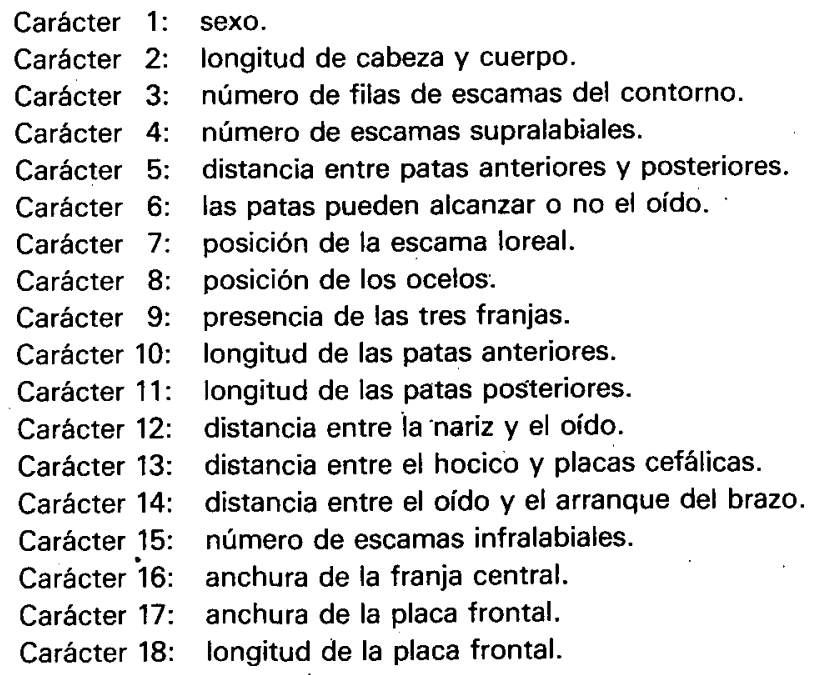


Para los cálculos se han empleado los datos del total de los 130 ejemplares.

La tabla 10 contiene la matriz de similaridades calculadas con el programa ANACAR. Los coeficientes señalados con un asterisco representan el mismo bloque de correlación que se obtuvo en el análisis factorial entre las variables numéricas.

Algunas de las restantes similaridades de la tabla 10 merecen una consideración más detenida. Destaca en primer lugar los altos valores que presenta el sexo con una gran mayoría de caracteres; de ellos, los señalados con dos asteriscos corresponden a correlaciones con variables numéricas de las que destacan el 0.29 con la longitud de las patas posteriores y el 0.25 con la distancia entre patas anteriores y posteriores. En general, los valores de similaridad con los atributos cuantitativos resultan exagerados por la inclusión de individuos inmaduros de pequeño tamaño. El dimorfismo sexual en relación con todas estas características se discutirá más adelante.

Los elevados valores de similaridad del sexo con caracteres no numéricos (coeficientes subrayados en la tabla 10 son en realidad información espúrea. Tanto en los tres caracteres de escamas (números $4,7 \mathrm{~V}$ 15) como en el de la presencia de un diseño de cinco bandas (carácter número 9), el falseamiento surge por existir estados muy poco frecuentes y que corresponden de forma asimétrica a machos o hembras. Son obvias también las correlaciones del carácter número 9 con los atributos numéricos (coeficientes señalados con dos asteriscos) ya que todas las formas juveniles carecen del diseño de las cinco bandas.

De mayor interés resultan los valores de similaridad entre los caracteres de escamas números 4,7 y 15. La comparación entre los dos primeros figura en la tabla 11 en que se puede apreciar claramente la correspondencia entre los estados $1-1$ y $4-4$ de ambos caracteres.

Por último, en la tabla 12 aparecen los valores de SUMRAT y SAMRAT de los dieciocho atributos considerados. Los mayores valores de ambos parámetros corresponden a los caracteres numéricos que comparten entre sí buena parte de su información, y a los caracteres numéricos 1 y 9 (sexo y presencia de diseño de cinco bandas). Destacan como caracteres de poco valor sistemático, el número de filas de escamas del contorno, la longitud relativa de las patas anteriores en relación con la distancia al oído, y la presencia y disposición de los ocelos. 


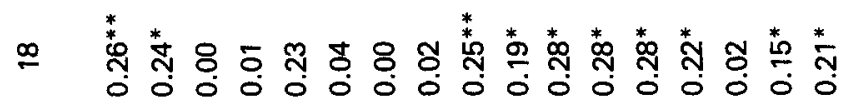

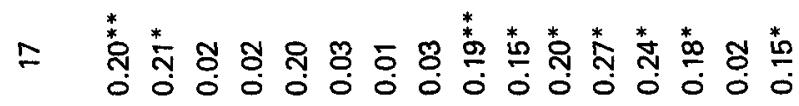

$88080 \%$

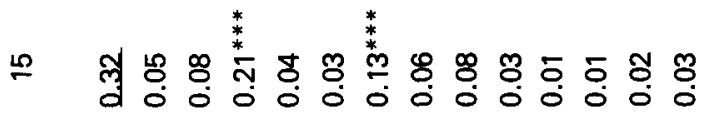

总

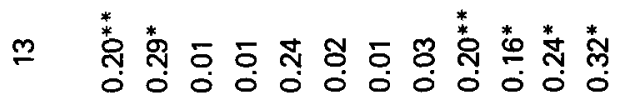

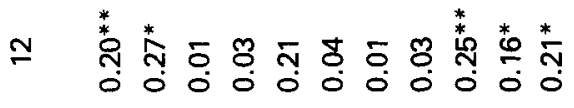

$=$ =

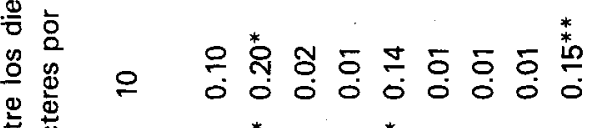

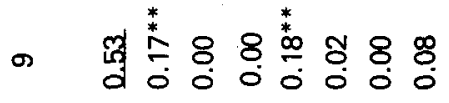

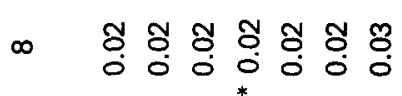

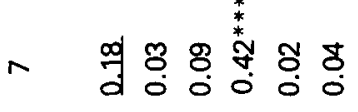

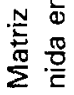

ำ 잉

密

-

m :

$\sim \stackrel{\frac{*}{0}}{\frac{*}{0}}$ 
TABLA 11: Comparación obtenida con el programa ANACAR de los caracteres 4 (número de escamas supralabiales) y 7 (posición de la escama loreal).

Carácter 4 (4) comparado con carácter 7 (4)

Número de objetos excluiidos de la comparación 0 .

Distancia entre caracteres 4 y $7 \quad D=.57963$

Similaridad $S=.42037$

Probabilidades de los estados del carácter 4.

(1) .18462 (2) 02308 (3) .03077 (4) .76154

Entropia incondicional del carácter $4=1.02929$

Matriz de probabilidades condicionales

$\begin{array}{cccccc} & 1 & 2 & 3 & 4 & \text { Entropía condicional } \\ 1 & .88462 & .00000 & .00000 & .11538 & .51595 \\ 2 & .33333 & .00000 & .00000 & .66667 & .91830 \\ 3 & .00000 & .00000 & .50000 & .50000 & 1.00000 \\ 4 & .00000 & .03093 & .02062 & .94845 & .34298\end{array}$

Información común a ambos caracteres $=.61822$

Entropia que queda en 4 después de observar $7=.41107$

Fracción de información del carácter 4 contenida en $7=.60063$

Probabilidades de los estados del carácter 7

(1) .200000 (2) 02308 (3).03077 (4) .74615

Eutropía incondicional del carácter $7=1.05961$

Matriz de probabilidades condicionales

$\begin{array}{cccccc} & 1 & 2 & 3 & 4 & \text { Entropia condicional } \\ 1 & .95833 & .04167 & .00000 & .00000 & .24988 \\ 2 & .00000 & .00000 & .00000 & 1.00000 & .00000 \\ 3 & .00000 & .00000 & .50000 & .50000 & 1.00000 \\ 4 & .03030 & .02020 & .02020 & .92929 & .47862\end{array}$

Información común a ambos caracteres $=.61822$

Entropia que queda en 7 después de observar $4=.44139$

Fracción de información del carácter 7 contenida en $4=.58344$ 
TABLA 12: Valores de SUMRAT y SAMRAT de los 18 caracteres considerados.

\begin{tabular}{|c|c|c|}
\hline & SUMRAT & SAMRAT \\
\hline Carácter $1 \ldots \ldots \ldots$ & 4.3658 & 5.8784 \\
\hline Carácter $2 \ldots \ldots \ldots$ & 5.1513 & 3.9539 \\
\hline Carácter $3 \ldots \ldots \ldots$ & 0.2857 & 0.8265 \\
\hline Carácter $4 \ldots . . \ldots \ldots$ & 1.3174 & 2.1125 \\
\hline Carácter $5 \ldots \ldots \ldots$ & 5.0666 & 3.7670 \\
\hline Carácter $6 \ldots \ldots \ldots$ & 1.0675 & 0.9163 \\
\hline Carácter $7 \ldots \ldots \ldots$ & 1.7944 & 1.9669 \\
\hline Carácter $8 \ldots \ldots \ldots$ & 0.9589 & 1.2913 \\
\hline Carácter $9 \ldots \ldots \ldots$ & 2.9902 & 7.7699 \\
\hline Carácter $10 \ldots \ldots \ldots$ & 3.7730 & 2.6530 \\
\hline Carácter $11 \ldots \ldots \ldots$ & 4.4145 & 3.9253 \\
\hline Carácter $12 \ldots \ldots \ldots$. & 5.0980 & 4.0855 \\
\hline Carácter $13 \ldots \ldots \ldots$ & 4.9441 & 4.0592 \\
\hline Carácter $14 \ldots \ldots \ldots$ & 4.5574 & 3.2263 \\
\hline Carácter $15 \ldots \ldots \ldots$ & 1.9993 & 2.4082 \\
\hline Carácter $16 \ldots \ldots \ldots$ & 1.6820 & 2.3764 \\
\hline Carácter $17 \ldots \ldots \ldots$ & 4.2513 & 4.0473 \\
\hline Carácter $18 \ldots \ldots \ldots$ & 4.5986 & 4.6800 \\
\hline
\end{tabular}

\subsubsection{Diagnosis subespecifica.}

La identificación a nivel de subespecie de los ejemplares de eslizón de Nueva Tabarca se llevó a cabo inicialmente por medio de una comparación entre las características de la población que figuran en las tablas 6 y 7 y las definiciones de VALVERDE (1966) que aparecen en la tabla 5 .

El número de escamas supralabiales anteriores a la subocular, el número de filas de escamas alrededor del cuerpo (si se entienden como un mínimo de 24 y un máximo de 26), y la presencia de manchas ocelares, inclinan la diagnosis hacia la subespecie bedriagai. No resulta tan acorde el hecho reseñado de que el diseño de las bandas no esté muy acusado ni tampoco el que las patas anteriores no alcancen el orificio auditivo, caracterísicas que en los eslizones de Nueva Tabarca se aproximan más a los dados como propios de la subespecie albaredae. 
Antes de iniciar el estudio de la correlación entre caracteres se pensó en el análisis canónico como la técnica más adecuada para la diferenciación subespecifica cuando se consideran en conjunto diferentes poblaciones (PETITPIERRE، 1973). Los resultados del análisis factorial, al poner de relieve la redundancia de los caracteres numéricos, únicos válidos en la aplicación del modelo canónico, descartaron la utilidad del método.

Al igual que en otros problemas taxonómicos, resulta difícil en este caso prescindir de variables no cuantitativas que aportan indudable información para la diferenciación sistemática. La irreductibilidad del carácter esencialmente mixto de los datos, lleva a considerar el análisis de coordenadas principales como el método más eficaz de ordenación (ESCARRE, 19721.

El citado método fue propuesto por GOWER (1966) y es una técnica de ordenación que parte de una matriz de similaridad de una cierta dimensión y a partir de ella genera un espacio euclídeo de menos dimensiones en el que se conserva una determinada función de las distancias originales. Su aplicación a problemas taxonómicos cuenta ya con una extensa bibliografía en la que son pioneros los trabajos de STEPHENSON y WILLIAMS (1968) y CLIFFORD, WILLIAMS y LANCE (1969).

La aplicación de esta técnica numérica al problema concreto de la subespeciación del eslizón ibérico, se hizo por medio del programa GOWER escrito en Fortran IV en el Departamento de Informática del C.E.U. de Alicante, y que utiliza la subrutina de diagonalización MATRIX. La compilación y ejecución del programa se realizó desde la terminal Univac Uniscope 100 del citado Centro.

En un primer análisis se utilizaron las once variables y los siete caracteres multiestados transformados en binarios. En el segundo y definitivo se transformaron los atributos numéricos en multiestados; se usó la misma matriz de datos de 18 caracteres multiestados que en el programa ANACAR, pero sólo se pudieron emplear 80 de los 130 individuos por problemas de capacidad de memoria en la unidad central del ordenador. Entre ellos estaban 33 no recogidos en Nueva Tabarca y 47 de la isla recogidos al azar.

El programa GOWER se inicia con el cálculo de la matriz de métricos de GOWER (1968) a la que se somete a un ajuste y centraje antes 
de la diagonalización. Tras obtener autovalores y autovectores (coordenadas) se tiene la opción de calcular correlaciones entre las variables originales, numéricas o binarias, y las coordenadas de los primeros cinco ejes; los cálculos los llevan a cabo las subrutinas CORRI y POINT, esta última de la colección de IBM.

Los primeros resultados obtenidos con el análisis de coordenadas principales vienen en la tabla 13. Para cada uno de los cinco primeros ejes se da el autovalor, el porcentaje de la variabilidad total, y el porcentaje acumulado. Los tres primeros ejes suman algo más de la mitad de la variabilidad, lo que representa una eficiencia aceptable en la reducción del espacio original.

Para la interpretación de los ejes se utilizó en primer lugar, y a modo de simple orientación, los cálculos de correlaciones entre coordenadas y los atributos originales numéricos, lo que resultó de gran utilidad para el primer eje. En la interpretación del segundo se ha seguido el método gráfico que utiliza GOLDSMITH (1973). No se ha llevado a cabo una delimitación formal de los grupos más homogéneosque resultan de la ordenación, pero puede realizarse con las interesantes técnicas de clasificación politética divisiva propuestas por LAMBERT y Col. (1973) y NOY-MEIR (1973).

En la figura 5(a) aparecen los 80 individuos que se hicieron intervenir en el análisis representados en el espacio formado por los ejes 1 y 2. A simple vista pueden reconocerse tres grupos: uno en el extremo positivo del eje 1 formado por los ejemplares números 56,66 a 71 , y 111. Un segundo que aparece en la parte superior separado según el eje 2, y un tercer grupo central, más denso y definido en su parte inferior.

TABLA 13: Autovalores y porcentajes de variabilidad correspondientes a los cinco primeros ejes obtenidos en el análisis de coordenadas principales.

Eje Autovalor Porc. de variabil. Porc. acumulado

$\begin{array}{lrrr}1 & 6.87 & 26.19 & 26.19 \\ 2 & 4.42 & 16.84 & 43.03 \\ 3 & 2.16 & 8.23 & 51.26 \\ 4 & 1.56 & 5.95 & 57.21 \\ 5 & 1.15 & 4.38 & 61.59\end{array}$


El eje 1 está correlacionado negativamente con todas las variables numéricas como ocurre casi siempre que hay varias variables dependientes de la dimensión total. El primero de los grupos citados corresponde, pues, a formas juveniles y carece de interés sistemático.

El eje 2 es de mucha mayor utilidad para la diferenciación de subespecies. En las figuras 6(a), 6(b), 6(c) y 6(d) aparecen como un 1 los ejemplares que presentan ese estado del carácter y con una raya horizontal todos los demás.

Los caracteres números $4,7,15$ y 2 son los mejores para la distinción de los dos grupos que origina el eje 2 .

En la figura 6(a) se representa el carácter número 4 (número de escamas supralabiales) y el estado 1 corresponde a los individuos que tienen tres a cada lado. En la figura $6(\mathrm{~b})$ aparece el carácter número 7 (escamas con las que contacta la loreal) y el estado 1 es el de los ejemplares en que a derecha e izquierda contacta únicamente con la segunda escama supralabial. La figura $6(\mathrm{c})$ corresponde al carácter número 15 (número de escamas infralabiales) en el que el estado 1 es el de los individuos con cuatro de dichas escamas en cada parte. En la figura 6(d) se ha representado el carácter: número de escamas del contorno, con el estado 1 para los casos en que dichas escamas son 24.

En la figura 5(b) aparecen únicamente los 33 ejemplares no directamente recolectados en Nueva Tabarca. En cada uno de ellos se indica la provincia a la que corresponde, a excepción de los ejemplares de Isla Plana pertenecientes a otras colecciones y que figuran con una raya.

El examen de estas figuras permite deducir que el eje 2 resulta válido para la separación de dos de las subespecies propuestas por VALVERDE (1966): el grupo superior correspondería al Chalcides bedriagai ssp. albaredae y el inferior al Chalcides bedriagai ssp. bedriagai. La distinción entre ambas subespecies que se desprende de los análisis numéricos, figura en la tabla 14, pero conviene tener presente la variabilidad indicada en la tabla 7.

En relación con la distribución geográfica coincide perfectamente lo propuesto por VALVERDE (1966) en los ejemplares de Tarragona, Castellón, Valencia y el de Alicante recolectado en el Mascarat, entre Altea y Calpe, es decir, en la zona norte de la provincia. Más problemáticos resultan los dos ejemplares de Nueva Tabarca y el de Murcia (Mar Menor) 


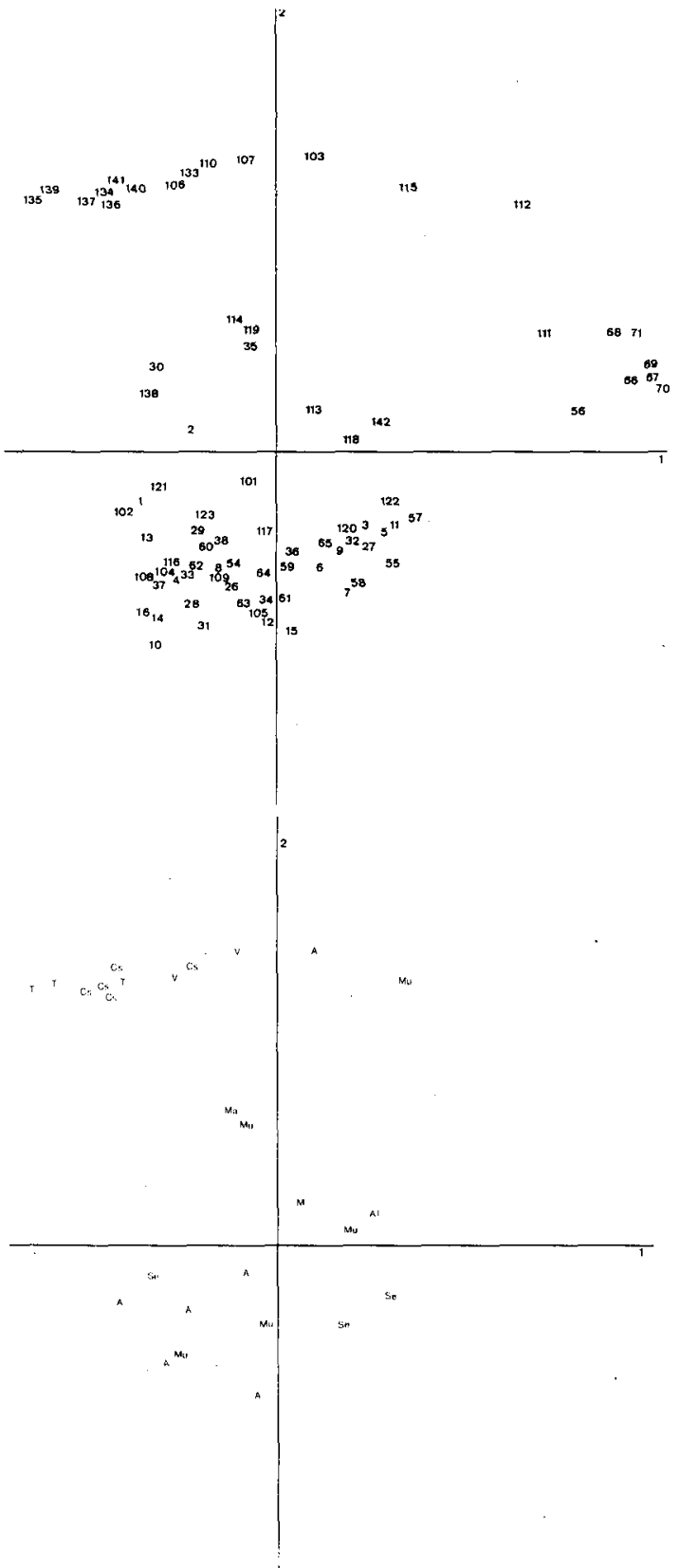

FIGURA 5. - Representación en el espacio formado por los ejes 1 y 2, de los 80 individuos de $\mathrm{Ch}$. bedriagai: a) General y b) no recolectados en N. Tabarca. 


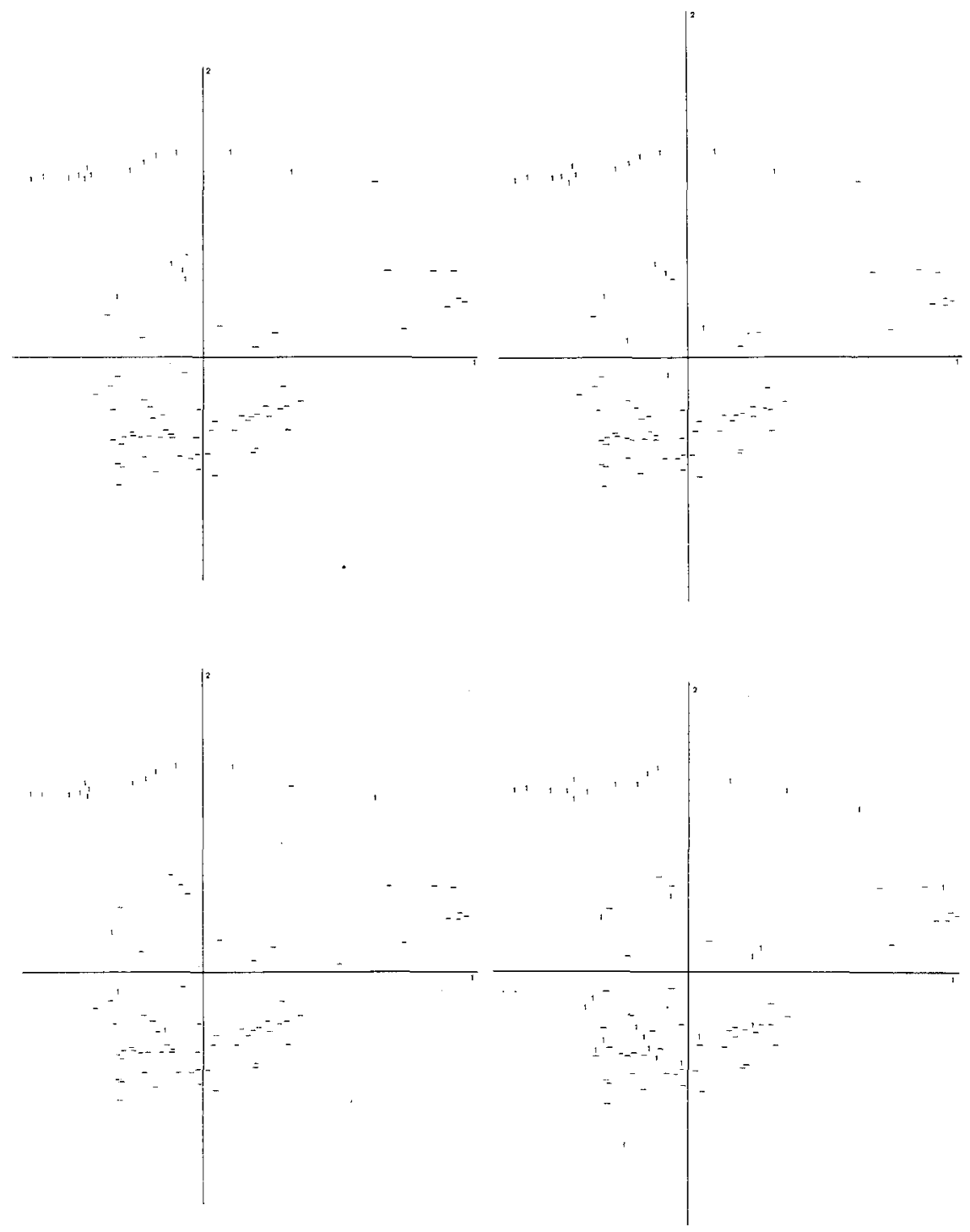

FIGURA 6. - La misma ordenación de la figura 5, pero representando con un 1 los ejemplares que poseen el estado 1 de los caracteres: a) núm. 4, número de escamas supralabiales; b) núm. 7, posición de la escama loreal; c) núm. 15, número de escamas infralabiales; d) núm. 3 , número dè escamas del contorno. 
que presentan caracteristicas de la ssp. albaredae, y que pertenecen todos ellos a la colección del Museo de Ciencias Naturales de Madrid, sobre todo el más próximo a los eslizones de Valencia y Castellón, y que sería un individuo excepcional en relación con todos los estudiados de la población insular. Conviene no descartar la posibilidad de que figuren en colección con localidad errónea.

Al no intervenir en el análisis más que un ejemplar de El Escorial, que por distribución geográfica pertenecería a la subespecie pistaciae y que queda en la zona periférica de la ssp. bedriagai, nada se puede añadir sobre su entidad y características diferenciales.

Persiste sin embargo un problema de nomenclatura con la subespecie típica, ya que si es válida la terra typica restricta dada por MERTENS y MULLER (1928), el tipo de la especie serian los ejemplares valencianos de la subespecie a/baredae que debiera entonces ser la típica. Por otra parte no son exactas las razones de VALVERDE para dar como típica la subespecie que difiere en más detalles de Chalcides ocellatus, ya que, según la definición que se ha conseguido en este estudio de la subespecie bedriagai, un ejemplar perteneciente a ella podría muy bien considerarse Chalcides ocellatus lloreal en contacto con las supralabiales 2 y 3 , y cuatro supralabiales en la figura) por los caracteres que MERTENS da en su clave, menos por el número de escamas del contorno.

\subsection{ESTUDIO ECOLOGICO.}

\subsubsection{Biotopo.}

Durante las primeras excursiones en busca de Chalcides bedriagai, se pensó que la población estaba prácticamente restringida a una vasta zona que rodea la ruinosa casa de campo del centro de la isla y una ancha franja central frente al cuartel de la Guardia Civil, cubierta gran parte del año por avena. Si bien la mayor densidad se halla en esta zona, más tarde se pudo comprobar por visualización y por datos que dieron los pobladores, que la población de eslizón ibérico ocupa toda Nueva Tabarca, recorriendo hasta los lugares más cercanos al poblado; no se tienen datos de los pequeños islotes que la circundan.

En aquella zona de mayor abundancia, predomina la vegetación con Mesembryanthemum crystallinum, Mesembryanthemum nodiflorum, As- 
TABLA 14: Distinción de subespeciès que se desprende del análisis numérico.

\begin{tabular}{|c|c|c|}
\hline & $\begin{array}{l}\text { Ch. bedriagai } \\
\text { ssp. bedriagai }\end{array}$ & $\begin{array}{l}\text { Ch. bedriagai } \\
\text { ssp. albaredae }\end{array}$ \\
\hline $\begin{array}{l}\text { Número de escamas supra- } \\
\text { labiales } \ldots \ldots \ldots \ldots \ldots \ldots\end{array}$ & cuatro & tres \\
\hline $\begin{array}{l}\text { La escama loreal contacta } \\
\text { con las supralabiales nú- } \\
\text { meros } \ldots \ldots \ldots \ldots \ldots \ldots\end{array}$ & dos y tres & dos \\
\hline $\begin{array}{l}\text { Número de escamas infra- } \\
\text { labiales } \ldots \ldots \ldots \ldots \ldots\end{array}$ & cinco & cuatro \\
\hline $\begin{array}{c}\text { Número de escamas del } \\
\text { contorno............ }\end{array}$ & $25-26(24)$ & 24 \\
\hline LCC media . . . . . . . . . . & 57.00 & 64.50 \\
\hline
\end{tabular}

teriscus maritimus, Convolvulus arvensis, Avena sterilis, Atriplex glauca y Suaeda fruticosa entre las dominantes, con una cobertura total en primavera de un 95 por ciento y una altura media de unos 15 a $20 \mathrm{cms}$. En otoño, esta vegetación decrece mucho, siendo la cobertura entonces de un 40 a 45 por ciento.

El terreno no es demasiado arenoso, y presenta pequeños cantos de 4 a $20 \mathrm{cms}$ y otros mayores de hasta $70 \mathrm{cms}$ muy cercanos a la casa en ruinas.

Debajo de cada piedra se encuentran en bastante cantidad Forficula auricularia, Formica sp., Porcellio sp., Micrositus gibbulus, Micrositus obesus, Cymindis lineola, Anthicus bifasciatus, Tisanuros, larvas y orugas, presas todas ellas muy comunes excepto los Tisanuros, del eslizón ibérico. Las piedras bajo las que se cobijan los eslizones, no suelen contener apenas fauna lapidícola.

Según SALVADOR (1974), las horas de captura para alimentación son las primeras de la mañana, refugiándose prácticamente el resto del 
día. Suelen hacerlo bajo las piedras planas que encierran debajo de eflas un alto grado de humedad y de un orden de 150 centímetros cuadrados de superficie. La probabilidad de encontrarlos debajo de piedras de estas características es de una de cada tres.

Un pequeño recuento de eslizones hallados bajo estas grandes piedras arrojó los siguientes datos, en inventarios de 4 metros cuadrados:

\begin{tabular}{|c|c|c|c|}
\hline Inventario & Núm. piedras & Dimensiones & Núm. eslizones \\
\hline 1 & 2 & $\begin{array}{l}55 \times 40 \ldots \ldots \\
25 \times 14\end{array}$ & 2 \\
\hline 2 & 2 & $\begin{array}{l}60 \times 50 \ldots \ldots \\
35 \times 30\end{array}$ & 1 \\
\hline 3 & 2 & $\begin{array}{l}70 \times 45 \ldots . \\
25 \times 20\end{array}$ & 1 \\
\hline 4 & 1 & $45 \times 40 \ldots \ldots$ & 0 \\
\hline 5 & 3 & $\begin{array}{l}70 \times 55 \ldots \ldots \\
50 \times 50\end{array}$ & 3 \\
\hline
\end{tabular}

3.2.2. Estructura de la población.

Aunque BOSCA (1880) reseña ya que en el eslizón ibérico se cumple la regla general en los saurios de que las hembras tienen el cuerpo más largo que los machos, la diferenciación de los sexos en los ejemplares de Nueva Tabarca se abordó con la inspección de los genitales. En los numerosos individuos recolectados en julio de 1972 no se encontraron huevos, ni células espermáticas en la observación microscópica del supuesto tejido testicular. Unos pocos eslizones recolectados a finales de abril de 1974 confirmaron la diferenciación: dos de las hembras tenían huevos, una de ellas cuatro que median entre 4 y $5 \mathrm{~mm}$, mientras 
que en la otra eran de 2-3 mm; en un frotis de tejido genital de un supuesto macho se pudieron observar espermatozoides.

En el análisis de caracteres se ha visto la existencia de correlación entre sexo y bastantes atributos numéricos. Entre ellos, la distancia entre patas anteriores y posteriores es la que presenta mayor valor de similaridad con el sexo y es, presumiblemente, el mejor carácter numérico para la diferenciación. Para el conjunto de los machos el valor medio del carácter es de $39.32 \mathrm{~mm}$ y la desviación típica vale 3.69; en las hembras, la media es de 45.02 y la desviación típica vale 4.71 . La $t$ de Student de la comparación de las medias vale 5.85 y es significativa a nivel de $\mathrm{p} \quad 0.01$. Para el carácter de la longitud de las patas posteriores, las medias de hembras y machos son respectivamente 13.42 y $13.11 \mathrm{~mm}$ y las desviaciones típicas 1.24 y 1.00 . El valor para la $t$ de Student es de 1.29 significativo a nivel de $p \quad 0.05$.

El porcentaje de sexos en la población de Nueva Tabarca es de un 53.57 por ciento de hembras y un 46.42 por ciento de machos.

El estudio de las clases de edad en la población se ha hecho en la amplia muestra recolectada en el mes de julio de 1972. Si se disponen los individuos por valores de longitud de cabeza y cuerpo, agrupados en intervalos de $2 \mathrm{~mm}$ se obtiene el diagrama que aparece en la figura 7(a). En ella pueden reconocerse cuatro generaciones cuyas clases centrales corresponderían respectivamente a $34.5,54.5,62.5$ y $70.5 \mathrm{~mm}$ de longitud. Cada generación puede definirse por un valor medio. En los individuos de menos de un año se toma el valor de todos ellos, pero en los de uno, dos o tres años sólo se median los de la clase central con los de la anterior o posterior. Los valores medios calculados son de 34.5, 54.2, 61.5 y 70.6 respectivamente. Si se representan estos puntos en el modelo logístico (figura $7(b)$ ) con la edad supuesta en abcisas y $\ln ((K-L) / L)$ en ordenadas, donde $K$ es el tamaño máximo y $L$ el valor de la longitud de cabeza y cuerpo, y en el supuesto inicial de que los individuos de la generación del año tienen 15 días, la recta que se construye presenta una pendiente de 0.95 y una ordenada en el origen de 0.124 . El valor del coeficiente de correlación que estima la bondad del ajuste es de 0.988 . Parece pues verosímil, el que la población esté verdaderamente formada por individuos de cuatro generaciones y puede admitirse una longevidad máxima de cuatro o cinco años.

Más complicado resulta intentar saber la edad de los individuos más jóvenes. SALVADOR (1974) da como longitud total de los recién naci- 

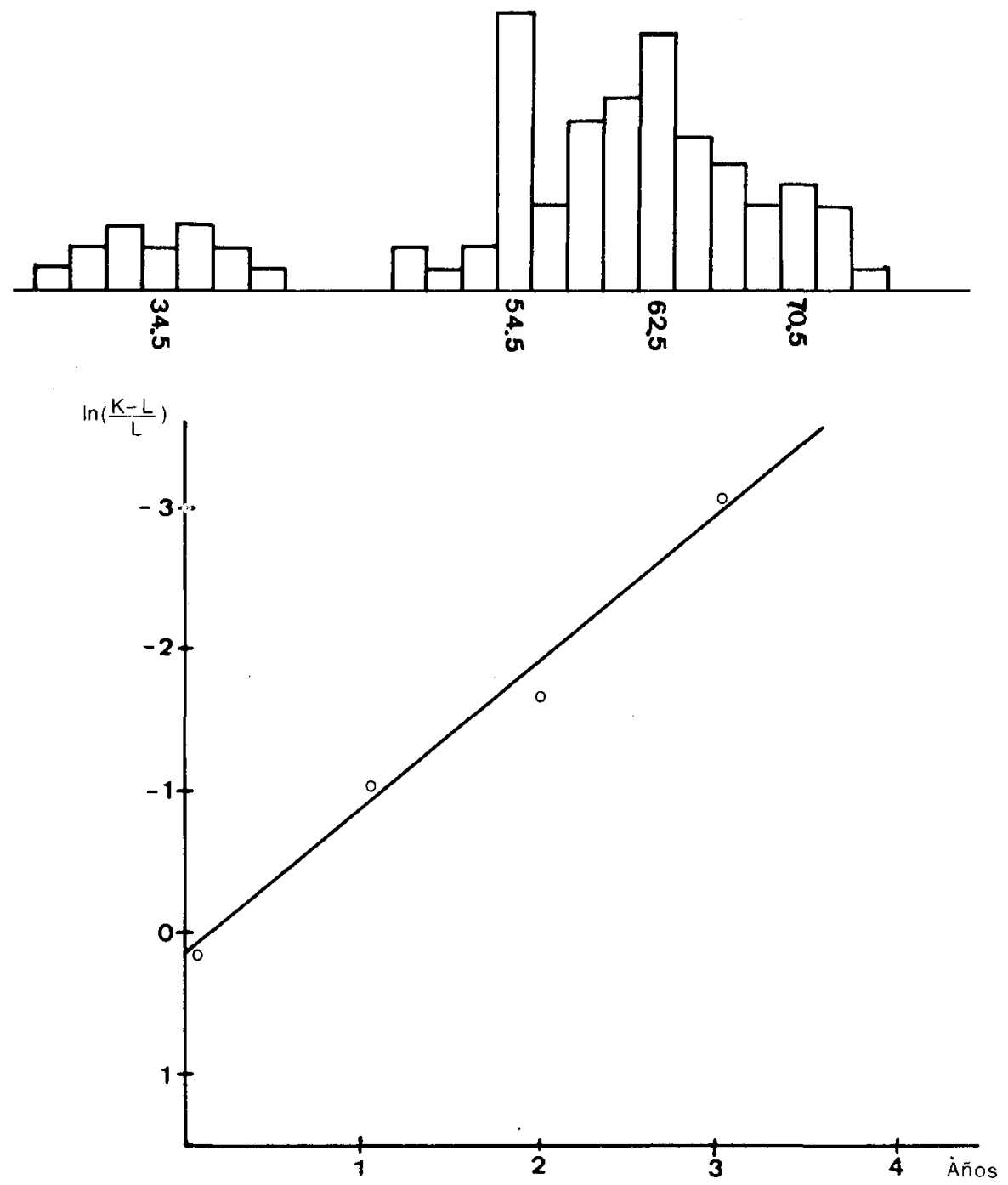

FIGURA 7. - a) Histograma de las LCC con intervalos de $2 \mathrm{~mm}$ de la muestra de eslizones recolectada en julio de 1972; b) Regresión según el modelo logístico entre crecimiento $y$ edad. 
dos $54 \mathrm{~mm}$ y como fecha de la puesta la de septiembre. El dato de la longitud citado representa una longitud de cabeza y cuerpo de 25.87 milimetros ya que en los ejemplares jóvenes la relación LCC/longitud cola, es igual a 0.92. Si se admite este valor de longitud y según el modelo logístico, los individuos juveniles de Nueva Tabarca tendrían seis meses y habrían nacido en el mes de enero, cosas ambas que parecen muy improbables.

Si se piensa en el tamaño de los huevos de las hembras recolectadas el 22 de abril de 1974, y en el dato aportado por BOSCA (1880) de una hembra con huevos del tamaño de un guisante, de finales de mayo, parece más probable que hacia finales de este mes y durante todo junio sea la época de aparición de las crías, en cuyo caso los individuos de la primera generación tendrán alrededor de un mes y medio. Para esta edad la LCC que correspondería al momento de nacer según la regresión sería de $32.3 \mathrm{~mm}$.

\subsubsection{Alimentación. Análisis de contenidos estomacales.}

El estudio del régimen alimenticio del eslizón ibérico de Nueva Tabarca se ha obtenido de un exhaustivo análisis de todo su aparato digestivo. Mediante una pequeña muestra de la fauna lapidícola y fitófila de la isla, la identificación mediante lupa binocular ha dado un total de 338 presas repartidas entre 17 especies y diversos huevos de un tamaño de $2-3 \mathrm{~mm}$.

Las presas, ordenadas según los tamaños crecientes, son:

1: Anthicus bifasciatus (Fam. Anthicidae, $2.1 \mathrm{~mm}$ ).

2: Aethus laticollis (Fam. Cydnidae, $4.3 \mathrm{~mm}$ ).

3: Restos de Arácnidos $(4.5-7.0 \mathrm{~mm})$.

4: Sitona humeralis (Fam. Curculionidae, $5.5 \mathrm{~mm}$ ).

5: Formica sp. (Fam. Formicidae, $6.0 \mathrm{~mm}$ ).

6: Phytonomus fasciculatus (Fam. Curculionidae, $6.2 \mathrm{~mm}$ ).

7: Masoreus wetterhalli (Fam. Masoreidae, $6.4 \mathrm{~mm}$ ).

8: Liorhyssus hialinus (Fam. Rhopolidae, $7.2 \mathrm{~mm}$ ).

9: Micrositus obesus (Fam. Tenebrionidae, $9.1 \mathrm{~mm}$ ).

10: Cymindis lineola (Fam. Lebiidae, $10.4 \mathrm{~mm}$ ).

11: Harpalus tenebrosus (Fam. Harpalidae, $10.4 \mathrm{~mm}$ ).

12: Centrocoris variegatus (Fam. Coreidae, $11.2 \mathrm{~mm}$ ). 
13: Micrositus gibbulus (Fam. Tenebrionidae, $13.0 \mathrm{~mm}$ ).

14: Forficula auricularia (Fam. Forficulidae, $18.0 \mathrm{~mm}$ ).

15: Porcellio sp. (Ord. Isópodos, $23.0 \mathrm{~mm}$ ).

16: Oruga tipo Pimelia (incl. larvas de Orius, hasta $24 \mathrm{~mm}$ ).

17: Oruga tipo Tentyria (hasta $25 \mathrm{~mm}$ ).

Todos estos datos quedan reunidos y resumidos en la matriz de alimentación de la tabla 15.

TABLA 15: Matriz de alimentación del eslizón en N. Tabarca.

PRESAS

$\begin{array}{llllllllllllllllll}\text { ESLIZONES } & 1 & 2 & 3 & 4 & 5 & 6 & 7 & 8 & 9 & 10 & 11 & 12 & 13 & 14 & 15 & 16 & 17\end{array}$

16 ind.

$28-48 \mathrm{~mm} \ldots \ldots \ldots \ldots . \quad 17172217-3-\ldots+1-1-2-$

24 ind.

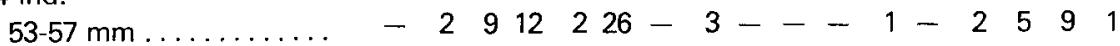

32 ind.

$58-66 \mathrm{~mm} \ldots \ldots \ldots \ldots . \quad 131536 \quad 13415 \ldots \ldots$

20 ind.

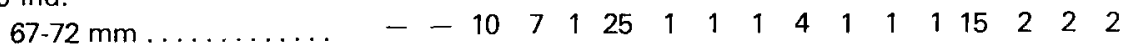

Las presas por otra parte pueden agruparse de acuerdo a su microbiotopo, o quizá mejor, en consonancia con el tipo de alimentación. Según datos de ANTOINE (1962) y PORTEVIN (1935) se pueden clasificar como sigue:

Fitófagos ................. Aethus laticollis

Centrocoris variegatus

Liorhyssus hialinus

Sitona humeralis

Phytonomus fasciculatus 
Carnívoros

Omnivoros

(detritívoro, granívoro)
Masoreus wetterhalli

Larva de Orius

Cymindis lineola

Harpalus tenebrosus

Formica sp.

Forficula auricularia

Orugas

Porcellio sp.

Anthicus bifasciatus

Micrositus obesus

Micrositus gibbulus

Los grupos carnívoro y omnívoro son por lo general lapidícolas y llenan este biotopo sublapídeo. El grupo herbívoro suele ocupar los 10 ó 15 primeros centímetros aéreos, en las partes inferiores de Suaeda y Atriplex (ANTOINE, 1962).

Otras presas frecuentes y adecuadas por el tamaño como son los Tisanuros, no aparecen en estómagos, bien por su agilidad en los movimientos (todos los que aparecen son insectos lentos) o bien por la excesiva fragilidad de sus anillos, muy sensibles a la digestión; tampoco aparecen coccinélidos que habitan zonas altas de vegetales que son completamente inaccesibles al eslizón ibérico.

Los moluscos, aun por su tamaño, no constituyen presa fácil, sin duda por el duro caparazón.

El régimen alimenticio viene acotado por el tamaño de las presas y por el grado de quitinización, ya que, ejemplares de esiizón de pocas dimensiones son capaces de tragar orugas de Pimelia de $20 \mathrm{~mm}$ y son incapaces, los más grandes, de engullir apenas ejemplares de Micrositus gibbulus $(13.0 \mathrm{~mm})$.

La información contenida en la matriz de alimentación original puede resumirse en una más sencilla, en la que las presas se agrupan por órdenes o clases tal como figura en la tabla 16, que permite hacer algunas comparaciones interesantes. Si con los datos que en ella figuran se calcula la diversidad de alimentación medida por la expresión de SHANNON (1948) se obtiene un valor de 2.35, que es de un orden de magnitud similar, aunque menor, al que resulta del cálculo con los datos de alimentación de VALVERDE (1967) que es de 2.80. Según cualquiera de 
TABLA 16: Matriz de alimentación simplificada de la población de eslizón de N. Tabarca.

\begin{tabular}{|c|c|c|}
\hline & Total & Porcentaje \\
\hline Arácnidos ........... & 51 & 15.08 \\
\hline Coleópteros . . . . . . . . & 160 & 47.33 \\
\hline Heterópteros . . . . . . . & 27 & 7.98 \\
\hline Himenópteros ......... & 5 & 1.47 \\
\hline Dermápteros . . . . . . . . & 26 & 7.69 \\
\hline Isópodos.............. & 15 & 4.43 \\
\hline Larvas .............. & 31 & 9.17 \\
\hline Huevos . . . . . . . . . . & 23 & 6.80 \\
\hline
\end{tabular}

las dos medidas, Chalcides bedriagai sería considerada como una especie eurífaga según los límites propuestos por BARBAULT (1974) para los escíndidos de las sabanas de Lamto, en las que distingue tres especies eurífagas con valores de diversidad comprendidos entre 2.45 y 2.52 , una intermedia con diversidad de 1.80 y otra claramente estenófaga con una diversidad de sólo 1.15 .

En la taxocenosis de que forma parte el eslizón sólo tiene como posibles competidores tróficos, en Nueva Tabarca, las dos salamanquesas. Hemidactylus turcicus es muy escaso y sólo se han podido obtener datos de alimentación de dos ejemplares muy jóvenes. Tarentola mauritanica es bastante más abundante y para ella se ha podido construir la matriz de alimentación que figura en la tabla 17. Los análisis de contenidos del tubo digestivo son insuficientes para intentar una cuantificación de la intensidad de la competencia, pero se puede señalar que a excepción de unos pequeños blátidos y de un locústido, todas las demás presas forman parte también de la dieta del eslizón.

Conociendo ya la base alimenticia de Chalcides bedriagai, para completar la red trófica centrada en él, diremos que Malpolon monspessulanus, que ocupa el vértice de la pirámide trófica de la isla, y los gatos que deambulan por la isla (según datos de los pobladores) son predadores del eslizón. En cuanto a otros posibles predadores como las aves carnívoras Larus argentatus, Larus ridibundus, Laniur senator y Falco peregrinus, se carece de datos precisos (figura 8 ). 


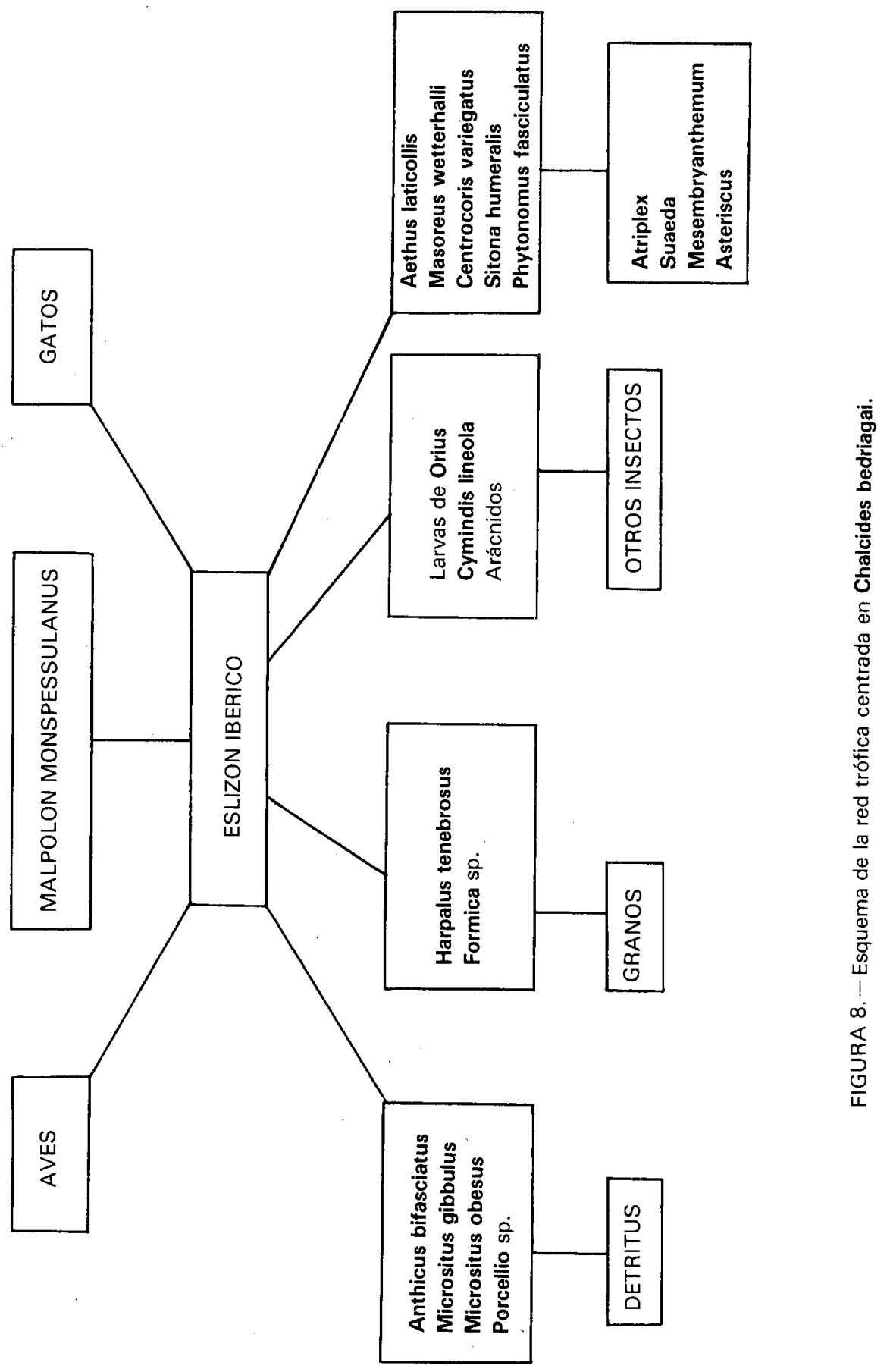


TABLA 17: Matriz de alimentación simplificada de Tarentola mauritanica en N. Tabarca.

NUMERO DE INDIVIDUO

\begin{tabular}{|c|c|c|c|c|c|c|c|c|}
\hline & 1 & 2 & 3 & 4 & 5 & 6 & 7 & 8 \\
\hline Porcellio sp. & & 1 & 2 & 1 & & & & \\
\hline Arácnidos. . & 1 & & & & & 1 & 1 & \\
\hline Blátidos............ & & & 1 & & & & & 11 \\
\hline Locústidos $\ldots \ldots \ldots \ldots$ & & & & & 1 & & & \\
\hline Forficula auricularia....... & 3 & & 1 & 2 & & & 2 & 2 \\
\hline Formícidos ............. & & & & & & 7 & & \\
\hline Liorhyssus hialinus . & & & & & & & 1 & \\
\hline Larvas...$\ldots \ldots \ldots$ & & & & & 2 & & & \\
\hline Parásitos.......... & 4 & 18 & 5 & 2 & 2 & 2 & & 6 \\
\hline
\end{tabular}

\section{RESUMEN}

Este trabajo consiste en un estudio de algunos aspectos biológicos de la isla de N. Tabarca, especialmente de la población de Ch. bedriagai, el más abundante de sus vertebrados.

En primer lugar se reseñan datos sobre la climatología y geología de la isla; la flora, vegetación y fauna de la isla se estudian con cierto detaile.

A continuación se describe la posición taxonómica de $C h$. bedriagai y sus subespecies. Se hace un análisis taximétrico de 18 caracteres numéricos y multiestados siguiendo dos caminos diferentes: análisis factorial en los caracteres numéricos y análisis de caracteres en los de multiestado. Diez de los once atributos numéricos muestran una mutua y estrecha correlación y se relacionan también algunos otros caracteres de escamas. El método de análisis de coordenadas se emplea en la diferenciación de subespecies y los resultados concuerdan con la distinción hecha por VALVERDE (1966) entre Ch. bedriagai bedriagai y Ch. bedriagai albaredae.

En el estudio ecológico de este eridemismo ibérico se describe este biotopo insular. La distribución de edades muestra cuatro clases de 
acuerdo con el modelo logístico de crecimiento. También se tratan las proporciones de sexos y el dimorfismo. Las costumbres alimenticias de Ch. bedriagai en su medio insular se estudian con detalle. El análisis de un centenar de contenidos estomacales muestra 17 tipos de presas diferentes, de tamaños comprendidos entre 5 y $25 \mathrm{~mm}$ de longitud, predominando Coleópteros y Arácnidos. Finalmente se esboza una red trófica centrada en $\mathrm{Ch}$. bedriagai.

\section{SUMMARY}

This study constitutes an approach about some biological aspects of $\mathrm{N}$. Tabarca island, specially on the population of $\mathrm{Ch}$. bedriagai, the most abundant vertebrate.

Firts of all the geology and climatology of the island are reviewed and the flora, vegetation and fauna studied with some detail.

The taxonomic status of $C h$. bedriagai and his subespecies are described. A taximetric analysis of 18 defined numerical and multistate characters is made with two different strategies: a) factor analysis in the numerical characters and b) character analysis in the multistate ones. Ten of the eleven numerical atributes shows a mutual and strong correlation, and some other characters of scales are also correlated. The principal coordinated analysis method is employed in the subespecies differentiation and the results agree very much with the distinction made by VALVERDE (1966) between $C h$. bedriagai bedriagai and Ch. bedriagai albaredae.

In the ecological approach of this iberian endemism, the concrete insular biotope is described. The age structure shows four age classes according with the logistic model of growth. The sexual proportions and dimorphism are also dealed. The feeding habits of $C h$. bedriagai in this insular environment are studied with some detail. The analysis of a hundred stomacal contents give 17 differents preys between 5 and $25 \mathrm{~mm}$. long, with the predominante of Coleoptheraus and Arachnids. Finally an outline of a food web centered in Ch. bedriagai is schematiced. 


\section{BIBLIOGRAFIA CITADA}

ANONIMO. - Boletines mensuales del Servicio Meteorológico Nacional. Años 19451971.

ANTOINE, M. (1957).-Coleoptères Carabiques du Maroc. Mem. Soc. Sci. Nat. et Phys. du Maroc. T. 1.

ANTOINE, M. (1959).-Coleoptères Carabiques du Maroc. Mem. Soc. Sci. Nat. et Phys. du Maroc. T. 2.

ANTOINE, M. (1961).-Coleoptères Carabiques du Maroc. Mem. Soc. Sci. Nat. et Phys. du Maroc. T. 3.

ANTOINE, M. (1962).-Coleoptères Carabiques du Maroc. Mem. Soc. Sci. Nat. et Phys. du Maroc. T. 4.

BALCELLS, E. (1963), - El Poblamiento Vegetal y Animal de las islas Medas. C.S.I.C. An. Inst. Est. Gerundenses del Patronato J. M. Quadrado. Vol. XVI, ps. 5-31.

BARBAULT, E. (1974).-Observations Ecologiques dans la savane de Lamto (Côte d'ivoire). Bull. Ecol. 5 (1): 7-25.

BARTOLI, L.; GOUNOT, M., y JACQUINET, J. C. (1967)._Climatologie et Bioclimatologie de la Tunisie Septentrionale. Ann. Inst. Nat. Rech. Agrom. Tunisie.

BOLOS, O. de (1957). - De Vegetatione Valentina, I, Collect. Bot. V: 527-596.

BOLOS, O. de (1967). - Comunidades Vegetales de las Comarcas próximas al litoral situadas entre los ríos Llobregat y Segura. Mem. Real Acad. Cien. Art. Barcelona. Vol. XXXVIII, núm. 1.

BOSCA, E. (1880).-Gongylus bedriagai, nueva subespecie de la Península Ibérica. An. Soc. Esp. Hist. Nat. Vol. 9: 495-503.

BOULENGER, E. G. (1887). - Cat. Liz. Brit. Mus. 3: 402.

BRAUN-BLANQUET, J.-BOLOS, O. de (1954). - Datos sobre las Comunidades Terofíticas de las Ilanuras del Ebro Medio. Collect. Bot. IV: 235-244; Comm. S.I.G.M.A. 123.

BRAUN-BLANQUET, J.-BOLOS, O. de (1957). - Les Groupements Vegetaux du Bas$\sin$ Moyen de l'Ebre et leur Dynamisme. An. Est. Exp. Aula Dei, V. 
CLIFFORD, H. T., WILLIAMS, W. T. y LANGE, G. N. (1969). - A Further Numerical Contribution to the Classification of the Poaceae. Aust. J. Bot. 17: 113-31.

CUADRAS, C. (1972).-Análisis Factorial. Series de Programación del Análisis Multivariante número 3. Laboratorio de Cálculo Fac. de Ciencias Univ. de Barcelona.

DOTTRENS, E. (1963). - Batracies et Reptiles d'Europe. Delachaux-Niesle, Neuchatel. 260 p.p.

ESCARRE, A (1972). - Essai d'Application des Methodes de la Taxonomie Numérique à l'étude d'una Chénaie dans la Vallée de Burunda (Navarre). Inv. Pesq. 36 (1): 7-14.

ESPAÑOL, F. (1965). - El Poblamiento Entomológico de la isla Plana o de Nueva Tabarca. Inst. Biol. Aplic. T. XXXIX. Barcelona.

ESTABROOK, G. F. (1967). - An Information Theory Model for Character Analysis. Taxon 16: 86-97.

FLEMING, H. S., y APPAN, S. G. (1971). - Program Charanal (Character Analysis) Software Documentation. GURC/MTF. Environmental Data Management Teams Doc. Núm. 2.

GOLDSMITH, F. B. (1973). - The Vegetation of Exposed Sea Cliffs at South Stack, Angesey, I. The Multivariate Approach. J. Ecol. 61: 787-818.

GOUNOT, M. (1969). - Methodes d'etude Quantitative de la Vegetation. Masson, París.

GOWER, J. C. (1966). - Some Distance Properties of Latent Root and Vector Methods Used in Multivariate Analisis. Biometrika. 53: 325.

GOWER, J. C. (1968).-A General Coefficient of Similarity and Some of its Properties. Roneo Rothansted. Exp. Stat, 16 pp.

HARMAN, H. H. (1967). - Modern Factor Analisis. 2 Ed. University of Chicago Press. Chicago.

KAMPSCHUUR, U., y SIMON, O. J. (1969). - Sur la Geologie de l'ille de Tabarca (Prov. d'Alicante. Esp.) et sa Position Tectonique dans la Zone Betique (Cordilleres Betiques). C. R. Som. S.G.F. 2: 37-38.

LAMBERT, J. M. (1972). - Theorical Models for Large-Scale Vegetation Survey en Mathematical Models in Ecology, Ed. J. N. R. Jeffers, Blackwell.

LAMBERT, J. M., MEACOCK, S. E., BARRS, J., y SMARTT, P. F. M. (1973). - Axor and Monit: Two New Polithetyc-divisive Strategies for Hierarchical Classification. Taxon 22 (2/3): 173-176. 
LEGENDRE, P., y ROGERS, J. (1972). - Characters and Clustering in Taxonomy: A Synthesis of two Taximetric Procedures. Taxon 21 (5/6): 567-606.

MONTENAT, C. (1973). - Les Formations Neogenes et Quaternaires du Levant Espagnol. These d'Etat presente a l'Universite de Paris Sud, Centre d'Orsay. París. p. 356.

MERTENS, R., y MULLER (1928):-ABH. Senckeb Nature Ges, 41. Frankfurt.

MERTENS, R., y WERMUTH, H. (1960). - Die Amphibien und Reptilien Europas. Verlag Waldemar Kramer. Frankfurt. M. 264 pp.

NOY-MEIR, I. (1973). - Divisive Polythetic Classification on Vegetation Data by Optimized Divission on Ordination Components. J. Ecol. Vol. 61: 750-60.

PETITPIERRE, E. (1973). - Estudios Sistemáticos Citogenéticos y Evolutivos sobre el Género Timarcha (Coleoptera, Chrysomelidae). Tesis Doctoral. Universidad de Barcelona.

PORTEVIN, G.(1935).-Histoire Naturelle des Coleopteres de France. T. N., París.

RIGUAL, A. (1972). - Flora y Vegetación de la Provincia de Alicante. Inst. de Est. Alicantinos. Alicante. 403 pp.

SALVADOR, A. (1974). - Guía de los Anfibios y Reptiles Españoles. I.C.O.N.A. Madrid, $282 \mathrm{pp}$.

SHANNON, C. E. (1948). - A Mathematical Theory of Communication. Bell Sistem Tech. J. Val. 27: 379-623.

SNEATH, P. H. A., y SOKAL, R. R. (1973). - Numerical Taxonomy. The Principies and Practice of Numerical Classification. Freeman, San Francisco.

STEPHENSON, W., y WILLIAMS, W. T. (1968). - Numerical Approaches to the Relations of Certain American Swimming Crabs (Crustacea: Portunidae). Proc. Unit. Stat. Mus. Smith. 124.

VALVERDE, J. A. (1966). - Notas sobre Vertebrados. II. Sobre las Subespecies de Chalcides bedriagai (Boscá 1880). Bol. R. Soc. Española Hist. Nat. (Biolog.) 64: 169-70.

VALVERDE, J. A. (1967). - Estructura de una Comunidad Mediterránea de Vertebrados Terrestres. C.S.I.C. Monografia de Ciencias Modernas núm. 716. 218 pp. 